\title{
The Beltrami equations and prime ends
}

\author{
Vladimir Gutlyanskit, Vladimir Ryazanov, \\ AND EDUARD YAKUBOV
}

\begin{abstract}
We first study the boundary behavior of ring $Q$-homeomorphisms in terms of Carathéodory's prime ends and then give criteria to the solvability of the Dirichlet problem for the degenerate Beltrami equation $\bar{\partial} f=\mu \partial f$ in arbitrary bounded finitely connected domains $D$ of the complex plane $\mathbb{C}$.
\end{abstract}

2010 MSC. 30C62, 30D40, 37E30, 35A16, 35A23, 35J46, 35J67, 35J70, 35J75, 35Q35.

Key words and phrases. Dirichlet problem, degenerate Beltrami equations, regular solutions, simply connected domains, pseudoregular and multi-valued solutions, finitely connected domains, tangent dilatations.

\section{Introduction}

Let $D$ be a domain in the complex plane $\mathbb{C}$ and let $\mu: D \rightarrow \mathbb{C}$ be a measurable function with $|\mu(z)|<1$ almost everywhere in $D$. We study the Beltrami equation

$$
f_{\bar{z}}=\mu(z) f_{z}
$$

where $f_{\bar{z}}=\bar{\partial} f=\left(f_{x}+i f_{y}\right) / 2, f_{z}=\partial f=\left(f_{x}-i f_{y}\right) / 2, z=x+i y$, and $f_{x}$ and $f_{y}$ are partial derivatives of $f$ in $x$ and $y$, correspondingly.

The classic Dirichlet problem in a Jordan domain $D$ for the uniformly elliptic Beltrami equation, i.e., when $|\mu(z)| \leq k<1$ a.e., is the problem on the existence of a continuous function $f: D \rightarrow \mathbb{C}$ such that

$$
\begin{cases}f_{\bar{z}}=\mu(z) \cdot f_{z}, & \text { for a.e. } z \in D, \\ \lim _{z \rightarrow \zeta} \operatorname{Re} f(z)=\varphi(\zeta), & \forall \zeta \in \partial D,\end{cases}
$$

for a prescribed continuous boundary function $\varphi$, has been studied long ago, see, e.g., $[2,52]$.

Received 1.03.2015 
The main goal of this work is to study the Dirichlet problem in an arbitrary bounded finitely connected domain $D \subset \mathbb{C}$ when the condition of uniform ellipticity of the Beltrami equations is replaced by the condition that $|\mu(z)|<1$ a.e.

The degeneracy of the ellipticity of the Beltrami equations will be controlled by the dilatation coefficient

$$
K_{\mu}(z)=\frac{1+|\mu(z)|}{1-|\mu(z)|}
$$

as well as by the more refined quantity

$$
K_{\mu}^{T}\left(z, z_{0}\right)=\frac{\left|1-\frac{\overline{z-z_{0}}}{z-z_{0}} \mu(z)\right|^{2}}{1-|\mu(z)|^{2}}
$$

that is called the tangent dilatation quotient of the Beltrami equation (1.1) with respect to $z_{0}$, see, e.g., $[1,10,23,34,37,41]$. This quantity takes into account not only the modulus of the complex coefficient $\mu$ but also its argument. Note that

$$
K_{\mu}^{-1}(z) \leqslant K_{\mu}^{T}\left(z, z_{0}\right) \leqslant K_{\mu}(z) \quad \forall z \in D \quad \forall z_{0} \in \mathbb{C} .
$$

Our research is based on the existence theorems of homeomorphic $W_{\text {loc }}^{1,1}$ solutions for the degenerate Beltrami equations and on the theory of prime ends by Carathéodory for such solutions.

New criteria of the existence of homeomorphic $W_{\mathrm{loc}}^{1,1}$ solutions for the degenerate Beltrami equations can be found, for instance, in [11,26], see also the references therein. The boundary behavior of $W_{\mathrm{loc}}^{1,1}$ homeomorphic solutions and the Dirichlet problem for degenerate Beltrami equations in Jordan domains have been studied, e.g., in [17-19,37]. Concerning the Carathéodory's theory of prime ends for the conformal mappings, we refer the reader to [4] and [5, Ch. 9].

Let $E_{D}$ denote the space of prime ends of the domain $D$ and let $\bar{D}_{P}=$ $D \cup E_{D}$ stand for the completion of the domain $D$ by its prime ends with the topology described in [5, Section 9.5]. From now on, the continuity of mappings $f: \bar{D}_{P} \rightarrow{\overline{D^{\prime}}}_{P}$ and the boundary functions $\varphi: E_{D} \rightarrow \mathbb{R}$ as functions of the prime end $P$ should be understood with respect to the given topology. Now the boundary condition is written as

$$
\lim _{n \rightarrow \infty} \operatorname{Re} f\left(z_{n}\right)=\varphi(P),
$$

where the limit is taken over all sequences of points $z_{n} \in D$ converging to the prime end $P$ of the domain $D$. Note that (1.6) is equivalent to the condition that

$$
\lim _{z \rightarrow P} \operatorname{Re} f(z)=\varphi(P)
$$


along any ways in $D$ going to the prime end $P$ of the domain $D$.

Remark 1.1. The Carathéodory topology can be described in terms of metrics. Really, as is known, every bounded finitely connected domain $D$ in $\mathbb{C}$ can be mapped by a conformal mapping $g_{0}$ onto a circular domain $D_{0}$ whose boundary consists of a finite collection of mutually disjoint circles and isolated points, see, e.g., Theorem V.6.2 in [9]. Due to the well-known Weierstrass theorem, isolated singular points of bounded conformal mappings are removable, see Theorem 1.2 in [5]. Hence, isolated points of $\partial D$ correspond to isolated points of $\partial D_{0}$ and vice versa.

The mapping theorem allows us to reduce the case to the Carathéodory theorem, see, e.g., Theorem 9.4 in [5] for simply connected domains. Thus, we have a natural one-to-one correspondence between points of $\partial D_{0}$ and prime ends of the domain $D$, see also Theorem 4.1 in [31]. Determine, in $\bar{D}_{P}$, the metric $\rho_{0}\left(p_{1}, p_{2}\right)=\left|\widetilde{g_{0}}\left(p_{1}\right)-\widetilde{g_{0}}\left(p_{2}\right)\right|$, where $\widetilde{g_{0}}$ is the extension of $g_{0}$ to $\bar{D}_{P}$ just mentioned. If $g_{*}$ is another conformal mapping of the domain $D$ on a circular domain $D_{*}$, then the corresponding metric $\rho_{*}\left(p_{1}, p_{2}\right)=\left|\widetilde{g_{*}}\left(p_{1}\right)-\widetilde{g_{*}}\left(p_{2}\right)\right|$ generates the same convergence in $\bar{D}_{P}$ as the metric $\rho_{0}$ because $g_{0} \circ g_{*}^{-1}$ is a conformal mapping between $D_{*}$ and $D_{0}$. The latter mapping can be extended to a homeomorphism between $\overline{D_{*}}$ and $\overline{D_{0}}$, see, e.g., Theorem V.6.1' in [9]. Consequently, the given metrics induce the same topology in the space $\bar{D}_{P}$.

This topology coincides with the topology of prime ends described in inner terms of the domain $D$ in Section 9.5 of [5]. Later on, we prefer to apply the description of the topology of prime ends in terms of the given metrics. We will explore the metrizability of $\bar{D}_{P}$. Note also that the space $\bar{D}_{P}$ for every bounded finitely connected domain $D$ in $\mathbb{C}$ with the given topology is compact because the closure of the circular domain $D_{0}$ is a compact space and, by construction, $\widetilde{g}_{0}: \bar{D}_{P} \rightarrow \overline{D_{0}}$ is a homeomorphism.

Applying the description of the topology of prime ends given in Section 9.5 of [5], we reduce the case of bounded finitely connected domains to Theorem 9.3 in [5] for simple connected domains and obtain the following statement.

Lemma 1.1. Each prime end $P$ of a bounded finitely connected domain $D$ in $\mathbb{C}$ contains a chain of cross-cuts $\sigma_{m}$ lying on circles $S\left(z_{0}, r_{m}\right)$ with $z_{0} \in \partial D$ and $r_{m} \rightarrow 0$ as $m \rightarrow \infty$.

Throughout this work, $B\left(z_{0}, r\right)=\left\{z \in \mathbb{C}:\left|z-z_{0}\right|<r\right\}, \mathbb{D}=B(0,1)$, $S\left(z_{0}, r\right)=\left\{z \in \mathbb{C}:\left|z-z_{0}\right|=r\right\}, R\left(z_{0}, r_{1}, r_{2}\right)=\left\{z \in \mathbb{C}: r_{1}<\left|z-z_{0}\right|<\right.$ $\left.r_{2}\right\}$. 


\section{Regular domains}

Recall the following topological notion. A domain $D \subset \mathbb{C}$ is said to be locally connected at a point $z_{0} \in \partial D$ if, for every neighborhood $U$ of the point $z_{0}$, there is a neighborhood $V \subseteq U$ of $z_{0}$ such that $V \cap D$ is connected. If this condition holds for all $z_{0} \in \partial D$, then $D$ is said to be locally connected on $\partial D$. For a domain that is locally connected on $\partial D$, there is a natural one-to-one correspondence between prime ends of $D$ and points of $\partial D$, moreover, the topology of prime ends coincides with the Euclidean topology. Note that every Jordan domain $D$ in $\mathbb{C}$ is locally connected on $\partial D$, see, e.g., [53, p. 66].

The (conformal) modulus of a family $\Gamma$ of paths $\gamma$ in $\mathbb{C}$ is the quantity

$$
M(\Gamma)=\inf _{\varrho \in \operatorname{adm} \Gamma} \int_{\mathbb{C}} \varrho^{2}(z) d m(z),
$$

where a Borel function $\varrho: \mathbb{C} \rightarrow[0, \infty]$ is admissible for $\Gamma$, write $\varrho \in$ $\operatorname{adm} \Gamma$, if

$$
\int_{\gamma} \varrho d s \geqslant 1 \quad \forall \gamma \in \Gamma .
$$

Here $s$ is a natural parameter of the arc length on $\gamma$.

Later on, given sets $A, B$ and $C$ in $\mathbb{C}, \Delta(A, B ; C)$ denotes a family of all paths $\gamma:[a, b] \rightarrow \mathbb{C}$ joining $A$ and $B$ in $C$, i.e. $\gamma(a) \in A, \gamma(b) \in B$ and $\gamma(t) \in C$ for all $t \in(a, b)$.

We say that $\partial D$ is weakly flat at a point $z_{0} \in \partial D$ if, for every neighborhood $U$ of the point $z_{0}$ and every number $P>0$, there exists a neighborhood $V \subset U$ of $z_{0}$ such that

$$
M(\Delta(E, F ; D)) \geqslant P
$$

for all continua $E$ and $F$ in $D$ intersecting $\partial U$ and $\partial V$. We say that $\partial D$ is weakly flat if it is weakly flat at each point $z_{0} \in \partial D$.

We also say that a point $z_{0} \in \partial D$ is strongly accessible if, for every neighborhood $U$ of the point $z_{0}$, there exist a compactum $E$ in $D$, a neighborhood $V \subset U$ of $z_{0}$ and a number $\delta>0$ such that

$$
M(\Delta(E, F ; D)) \geqslant \delta
$$

for all continua $F$ in $D$ intersecting $\partial U$ and $\partial V$. We say that $\partial D$ is strongly accessible if each point $z_{0} \in \partial D$ is strongly accessible.

It is easy to see that if a domain $D$ in $\mathbb{C}$ is weakly flat at a point $z_{0} \in \partial D$, then the point $z_{0}$ is strongly accessible from $D$. The following statement is fundamental, see, e.g., Lemma 5.1 in [21] or Lemma 3.15 in [26]. 
Lemma 2.1. If a domain $D$ in $\mathbb{C}$ is weakly flat at a point $z_{0} \in \partial D$, then $D$ is locally connected at $z_{0}$.

The notions of strong accessibility and weak flatness at boundary points of a domain in $\mathbb{C}$ defined in [20], see also [21,36], are localizations and generalizations of the corresponding notions introduced in [24, 25], cf. with the properties $P_{1}$ and $P_{2}$ by Väisälä in [51] and also with the quasiconformal accessibility and the quasiconformal flatness by Näkki in [29].

A domain $D \subset \mathbb{C}$ is called a quasiextremal distance domain, abbr. QED-domain, see [8], if

$$
M(\Delta(E, F ; \mathbb{C}) \leqslant K \cdot M(\Delta(E, F ; D))
$$

for some $K \geqslant 1$ and all pairs of nonintersecting continua $E$ and $F$ in $D$.

It is well known, see, e.g., Theorem 10.12 in [51], that

$$
M(\Delta(E, F ; \mathbb{C})) \geqslant \frac{2}{\pi} \log \frac{R}{r}
$$

for any sets $E$ and $F$ in $\mathbb{C}$ intersecting all the circles $S\left(z_{0}, \rho\right), \rho \in(r, R)$. Hence, a QED-domain has a weakly flat boundary. An example in [26], Section 3.8, shows that the inverse conclusion is not true even in the case of simply connected domains in $\mathbb{C}$.

A domain $D \subset \mathbb{C}$ is called a uniform domain if each pair of points $z_{1}$ and $z_{2} \in D$ can be joined with a rectifiable curve $\gamma$ in $D$ such that

$$
s(\gamma) \leqslant a \cdot\left|z_{1}-z_{2}\right|
$$

and

$$
\min _{i=1,2} s\left(\gamma\left(z_{i}, z\right)\right) \leqslant b \cdot \operatorname{dist}(z, \partial D)
$$

for all $z \in \gamma$, where $\gamma\left(z_{i}, z\right)$ is the portion of $\gamma$ bounded by $z_{i}$ and $z$, see [28]. It is known that every uniform domain is a QED-domain but there exist QED-domains that are not uniform, see [8]. Bounded convex domains and bounded domains with smooth boundaries are simple examples of uniform domains and, consequently, QED-domains, as well as domains with weakly flat boundaries.

Recall that $\varphi: U \rightarrow \mathbb{C}$ is said to be a Lipschitz map provided $\mid \varphi\left(z_{1}\right)-$ $\varphi\left(z_{2}\right)|\leqslant M \cdot| z_{1}-z_{2} \mid$ for some $M<\infty$ and for all $z_{1}$ and $z_{2} \in U$, and a bi-Lipschitz map if, in addition, $M^{*}\left|z_{1}-z_{2}\right| \leqslant\left|\varphi\left(z_{1}\right)-\varphi\left(z_{2}\right)\right|$ for some $M^{*}>0$ and for all $z_{1}$ and $z_{2} \in U$. One says that $D$ in $\mathbb{C}$ is a Lipschitz domain if every point $z_{0} \in \partial D$ has a neighborhood $U$ that can be mapped by a bi-Lipschitz homeomorphism $\varphi$ onto the unit disk $\mathbb{D}$ in $\mathbb{C}$ 
such that $\varphi(\partial D \cap U)$ is the intersection of $\mathbb{D}$ with the real axis. Note that a bi-Lipschitz homeomorphism is quasiconformal and, consequently, the modulus is quasiinvariant under such a mapping. Hence, the Lipschitz domains have weakly flat boundaries.

\section{BMO, VMO, and FMO functions}

A real-valued function $u$ in a domain $D$ in $\mathbb{C}$ is said to be of bounded mean oscillation in $D$, abbr. $u \in \operatorname{BMO}(D)$, if $u \in L_{\text {loc }}^{1}(D)$ and

$$
\|u\|_{*}:=\sup _{B} \frac{1}{|B|} \int_{B}\left|u(z)-u_{B}\right| d m(z)<\infty,
$$

where the supremum is taken over all disks $B$ in $D, d m(z)$ corresponds to the Lebesgue measure in $\mathbb{C}$ and

$$
u_{B}=\frac{1}{|B|} \int_{B} u(z) d m(z) .
$$

We write $u \in \mathrm{BMO}_{\text {loc }}(D)$ if $u \in \operatorname{BMO}(U)$ for every relatively compact subdomain $U$ of $D$ (we also write $\mathrm{BMO}$ or $\mathrm{BMO}_{\text {loc }}$ if it is clear from the context what $D$ is).

The class BMO was introduced by John and Nirenberg (1961) in work [16] and soon became an important concept in harmonic analysis, partial differential equations, and related areas; see, e.g., [12,35].

A function $\varphi$ in BMO is said to have vanishing mean oscillation, abbr. $\varphi \in \mathrm{VMO}$, if the supremum in (3.1) taken over all balls $B$ in $D$ with $|B|<\varepsilon$ converges to 0 as $\varepsilon \rightarrow 0$. VMO has been introduced by Sarason in [49]. There are a number of works devoted to the study of partial differential equations with coefficients of the class VMO, see, e.g., $[6,15,27,32,33]$.

Remark 3.1. Note that $W^{1,2}(D) \subset V M O(D)$, see, e.g., [3].

Following [14], we say that a function $\varphi: D \rightarrow \mathbb{R}$ has finite mean oscillation at a point $z_{0} \in D$, abbr. $\varphi \in \operatorname{FMO}\left(z_{0}\right)$, if

$$
\varlimsup_{\varepsilon \rightarrow 0} f_{B\left(z_{0}, \varepsilon\right)}\left|\varphi(z)-\widetilde{\varphi}_{\varepsilon}\left(z_{0}\right)\right| d m(z)<\infty,
$$

where

$$
\widetilde{\varphi}_{\varepsilon}\left(z_{0}\right)=f_{B\left(z_{0}, \varepsilon\right)} \varphi(z) d m(z)
$$


is the mean value of the function $\varphi(z)$ over the disk $B\left(z_{0}, \varepsilon\right)$. Note that condition (3.2) includes the assumption that $\varphi$ is integrable in some neighborhood of the point $z_{0}$. We say also that a function $\varphi: D \rightarrow \mathbb{R}$ is of finite mean oscillation in $D$, abbr. $\varphi \in \operatorname{FMO}(D)$ or simply $\varphi \in \mathrm{FMO}$, if $\varphi \in \operatorname{FMO}\left(z_{0}\right)$ for all points $z_{0} \in D$. We write $\varphi \in \operatorname{FMO}(\bar{D})$ if $\varphi$ is given in a domain $G$ in $\mathbb{C}$ such that $\bar{D} \subset G$ and $\varphi \in \operatorname{FMO}(G)$.

The following statement is obvious by the triangle inequality.

Proposition 3.1. If, for a collection of numbers $\varphi_{\varepsilon} \in \mathbb{R}, \varepsilon \in\left(0, \varepsilon_{0}\right]$,

$$
\varlimsup_{\varepsilon \rightarrow 0} f_{B\left(z_{0}, \varepsilon\right)}\left|\varphi(z)-\varphi_{\varepsilon}\right| d m(z)<\infty,
$$

then $\varphi$ is of finite mean oscillation at $z_{0}$.

In particular, choosing here $\varphi_{\varepsilon} \equiv 0, \varepsilon \in\left(0, \varepsilon_{0}\right]$, we obtain the following.

Corollary 3.1. If, for a point $z_{0} \in D$,

$$
\varlimsup_{\varepsilon \rightarrow 0} f_{B\left(z_{0}, \varepsilon\right)}|\varphi(z)| d m(z)<\infty,
$$

then $\varphi$ has finite mean oscillation at $z_{0}$.

Recall that a point $z_{0} \in D$ is called a Lebesgue point of a function $\varphi: D \rightarrow \mathbb{R}$ if $\varphi$ is integrable in a neighborhood of $z_{0}$ and

$$
\lim _{\varepsilon \rightarrow 0} f_{B\left(z_{0}, \varepsilon\right)}\left|\varphi(z)-\varphi\left(z_{0}\right)\right| d m(z)=0 .
$$

It is known that almost every point in $D$ is a Lebesgue point for every function $\varphi \in L^{1}(D)$. Thus, we have the following corollary by Proposition 3.1.

Corollary 3.2. Every locally integrable function $\varphi: D \rightarrow \mathbb{R}$ has a finite mean oscillation at almost every point in $D$.

Remark 3.2. Note that the function $\varphi(z)=\log (1 /|z|)$ belongs to BMO in the unit disk $\Delta$, see, e.g., [35, p. 5], and hence also to FMO. However, $\widetilde{\varphi}_{\varepsilon}(0) \rightarrow \infty$ as $\varepsilon \rightarrow 0$, showing that condition (3.5) is only sufficient but not necessary for a function $\varphi$ to be of finite mean oscillation at $z_{0}$. Clearly, $\operatorname{BMO}(D) \subset \operatorname{BMO}_{\text {loc }}(D) \subset \operatorname{FMO}(D)$ and, as well-known, $\mathrm{BMO}_{\text {loc }} \subset L_{\text {loc }}^{p}$ for all $p \in[1, \infty)$, see, e.g., [16,35]. However, FMO is not a subclass of $L_{\mathrm{loc}}^{p}$ for any $p>1$ but only of $L_{\mathrm{loc}}^{1}$. Thus, the class FMO is much more wide than $\mathrm{BMO}_{\text {loc }}$. 
Versions of the next lemma has been first proved for BMO in [40]. For FMO, see works $[14,36,42,43]$ and books $[11,26]$.

Lemma 3.1. Let $D$ be a domain in $\mathbb{C}$ and let $\varphi: D \rightarrow \mathbb{R}$ be a nonnegative function of the class $\operatorname{FMO}\left(z_{0}\right)$ for some $z_{0} \in D$. Then

$$
\int_{\varepsilon<\left|z-z_{0}\right|<\varepsilon_{0}} \frac{\varphi(z) d m(z)}{\left(\left|z-z_{0}\right| \log \frac{1}{\left|z-z_{0}\right|}\right)^{2}}=O\left(\log \log \frac{1}{\varepsilon}\right) \quad \text { as } \varepsilon \rightarrow 0
$$

for some $\varepsilon_{0} \in\left(0, \delta_{0}\right)$ where $\delta_{0}=\min \left(e^{-e}, d_{0}\right), d_{0}=\inf _{z \in \partial D}\left|z-z_{0}\right|$.

\section{Beltrami equations and ring $Q$-homeomorphisms}

The following notion was motivated by the ring definition of Gehring for quasiconformal mappings, see, e.g., [7], and it is closely relevant with the Beltrami equations. Given a domain $D$ in $\mathbb{C}$ and a Lebesgue measurable function $Q: \mathbb{C} \rightarrow(0, \infty)$, we say that a homeomorphism $f: D \rightarrow \overline{\mathbb{C}}$ is a ring $Q$-homeomorphism at a point $z_{0} \in \bar{D}$ if

$$
M\left(\Delta\left(f C_{1}, f C_{2} ; f D\right)\right) \leqslant \int_{A \cap D} Q(z) \cdot \eta^{2}\left(\left|z-z_{0}\right|\right) d m(z)
$$

for any ring $A=A\left(z_{0}, r_{1}, r_{2}\right)$ and arbitrary continua $C_{1}$ and $C_{2}$ in $D$ that belong to the different components of the complement of the ring $A$ in $\overline{\mathbb{C}}$ including $z_{0}$ and $\infty$, correspondingly, and for any Lebesgue measurable function $\eta:\left(r_{1}, r_{2}\right) \rightarrow[0, \infty]$ such that

$$
\int_{r_{1}}^{r_{2}} \eta(r) d r \geqslant 1
$$

The notion was first introduced at inner points of a domain $D$ in work [41]. The ring $Q$-homeomorphisms at boundary points of a domain $D$ have first been considered in works $[44,45]$.

By Lemma 2.2 in [38] or Lemma 7.4 in [26], we obtain the following criterion for homeomorphisms in $\mathbb{C}$ to be ring $Q$-homeomorphisms, see also Theorem A.7 in [26].

Lemma 4.1. Let $D$ and $D^{\prime}$ be bounded domains in $\mathbb{C}$ and $Q: \mathbb{C} \rightarrow(0, \infty)$ be a measurable function. A homeomorphism $f: D \rightarrow D^{\prime}$ is a ring $Q$ homeomorphism at $z_{0} \in \bar{D}$ if and only if

$M\left(\Delta\left(f S_{1}, f S_{2} ; f D\right)\right) \leqslant\left(\int_{r_{1}}^{r_{2}} \frac{d r}{\|Q\|\left(z_{0}, r\right)}\right)^{-1} \forall r_{1} \in\left(0, r_{2}\right), r_{2} \in\left(0, d_{0}\right)$ 
where $S_{i}=S\left(z_{0}, r_{i}\right), i=1,2, d_{0}=\sup _{z \in D}\left|z-z_{0}\right|$ and $\|Q\|\left(z_{0}, r\right)$ is the $L^{1}$-norm of $Q$ over $D \cap S\left(z_{0}, r\right)$.

By Theorem 4.1 in [37], every homeomorphic $W_{\text {loc }}^{1,1}$ solution of the Beltrami equation (1.1) in a domain $D \subseteq \mathbb{C}$ is the so-called lower $Q$ homeomorphism at every point $z_{0} \in \bar{D}$ with $Q(z)=K_{\mu}^{T}\left(z, z_{0}\right), z \in D$, and $Q(z) \equiv \varepsilon>0$ in $\mathbb{C} \backslash D$. On the other hand, by Theorem 2 in [18] for a locally integrable $Q$, if $f: D \rightarrow D^{\prime}$ is a lower $Q$-homeomorphism at a point $z_{0} \in \bar{D}$, then $f$ is a ring $Q$-homeomorphism at the point $z_{0}$. Thus, we have the following conclusion.

Theorem 4.1. Let $f$ be a homeomorphic $W_{\mathrm{loc}}^{1,1}$ solution of the Beltrami equation (1.1) in a domain $D \subseteq \mathbb{C}$ and $K_{\mu} \in L^{1}(D)$. Then $f$ is a ring $Q$-homeomorphism at every point $z_{0} \in \bar{D}$ with $Q(z)=K_{\mu}^{T}\left(z, z_{0}\right), z \in D$.

In fact, it is sufficient to assume here that $K_{\mu}^{T}\left(z, z_{0}\right)$ is integrable along the circles $\left|z-z_{0}\right|=r$ for a.e. small enough $r$, instead of the condition $K_{\mu} \in L^{1}(D)$.

\section{The continuous extension of ring $Q$-homeomorphisms}

Lemma 5.1. Let $D$ and $D^{\prime}$ be bounded finitely connected domains in $\mathbb{C}$ and let $f: D \rightarrow D^{\prime}$ be a ring $Q_{z_{0}}$-homeomorphism at every point $z_{0} \in \partial D$. Suppose that

$\int_{D\left(z_{0}, \varepsilon, \varepsilon_{0}\right)} Q_{z_{0}}(z) \cdot \psi_{z_{0}, \varepsilon, \varepsilon_{0}}^{2}\left(\left|z-z_{0}\right|\right) d m(z)=o\left(I_{z_{0}, \varepsilon_{0}}^{2}(\varepsilon)\right)$ as $\varepsilon \rightarrow 0 \forall z_{0} \in \partial D$

where $D\left(z_{0}, \varepsilon, \varepsilon_{0}\right)=\left\{z \in D: \varepsilon<\left|z-z_{0}\right|<\varepsilon_{0}\right\}$ for every small enough $0<\varepsilon_{0}<d\left(z_{0}\right)=\sup _{z \in D}\left|z-z_{0}\right|$ and where $\psi_{z_{0}, \varepsilon, \varepsilon_{0}}(t):(0, \infty) \rightarrow[0, \infty]$, $\varepsilon \in\left(0, \varepsilon_{0}\right)$, is a family of (Lebesgue) measurable functions such that

$$
0<I_{z_{0}, \varepsilon_{0}}(\varepsilon):=\int_{\varepsilon}^{\varepsilon_{0}} \psi_{z_{0}, \varepsilon, \varepsilon_{0}}(t) d t<\infty \quad \forall \varepsilon \in\left(0, \varepsilon_{0}\right) .
$$

Then $f$ can be extended to a continuous mapping of $\bar{D}_{P}$ onto ${\overline{D^{\prime}}}_{P}$.

Proof. By Remark 1.1, with no loss of generality, we may assume that $D^{\prime}$ is a circular domain and, thus, $\overline{D^{\prime}} P=\overline{D^{\prime}}$. Let us first prove that the cluster set

$$
L=C(P, f):=\left\{\zeta \in \mathbb{C}: \zeta=\lim _{n \rightarrow \infty} f\left(z_{n}\right), z_{n} \rightarrow P, z_{n} \in D, n=1,2, \ldots\right\}
$$


consists of a single point $\zeta_{0} \in \partial D^{\prime}$ for each prime end $P$ of the domain D.

Note that $L \neq \varnothing$ by compactness of the set $\overline{D^{\prime}}$, and $L$ is a subset of $\partial D^{\prime}$, see, e.g., Proposition 2.5 in [36] or Proposition 13.5 in [26]. Let us assume that there exist at least two points $\zeta_{0}$ and $\zeta_{*} \in L$. Set $U=$ $B\left(\zeta_{0}, \rho_{0}\right)=\left\{\zeta \in \mathbb{C}:\left|\zeta-\zeta_{0}\right|<\rho_{0}\right\}$, where $0<\rho_{0}<\left|\zeta_{*}-\zeta_{0}\right|$.

Let $\sigma_{k}, k=1,2, \ldots$, be a chain of cross-cuts of $D$ in the prime end $P$ lying on circles $S_{k}=S\left(z_{0}, r_{k}\right)$ from Lemma 1.1, where $z_{0} \in \partial D$. Let $D_{k}, k=1,2, \ldots$ be the domains associated with $\sigma_{k}$. Then there exist points $\zeta_{k}$ and $\zeta_{k}^{*}$ in the domains $D_{k}^{\prime}=f\left(D_{k}\right)$ such that $\left|\zeta_{0}-\zeta_{k}\right|<\rho_{0}$ and $\left|\zeta_{0}-\zeta_{k}^{*}\right|>\rho_{0}$ and, moreover, $\zeta_{k} \rightarrow \zeta_{0}$ and $\zeta_{k}^{*} \rightarrow \zeta_{*}$ as $k \rightarrow \infty$. Let $C_{k}$ be continuous curves joining $\zeta_{k}$ and $\zeta_{k}^{*}$ in $D_{k}^{\prime}$. Note that, by construction, $\partial U \cap C_{k} \neq \varnothing, k=1,2, \ldots$

By the condition of strong accessibility of the point $\zeta_{0}$, there are a continuum $E \subset D^{\prime}$ and a number $\delta>0$ such that

$$
M\left(\Delta\left(E, C_{k} ; D^{\prime}\right)\right) \geqslant \delta
$$

for all large enough $k$. Note that $C=f^{-1}(E)$ is a compact subset of $D$ and hence $d_{0}=\operatorname{dist}\left(z_{0}, C\right)>0$. Let $\varepsilon_{0} \in\left(0, d_{0}\right)$ be small enough from the hypotheses of the lemma. With no loss of generality, we may assume that $r_{k}<\varepsilon_{0}$ and (5.2) holds for all $k=1,2, \ldots$.

Let $\Gamma_{m}$ be a family of all continuous curves in $D \backslash D_{m}$ joining the circle $S_{0}=S\left(z_{0}, \varepsilon_{0}\right)$ and $\overline{\sigma_{m}}, m=1,2, \ldots$ Note that, by construction, $C_{k} \subset D_{k}^{\prime} \subset D_{m}^{\prime}$ for all $k \geqslant m$ and, thus, by the principle of minorization, $M\left(f\left(\Gamma_{m}\right)\right) \geqslant \delta$ for all $m=1,2, \ldots$.

On the other hand, every function

$$
\eta(t)=\eta_{m}(t):=\psi_{z_{0}, r_{m}, \varepsilon_{0}}^{*}(t) / I_{z_{0}, \varepsilon_{0}}\left(r_{m}\right), \quad m=1,2, \ldots,
$$

satisfies condition (4.2) and hence

$$
M\left(f \Gamma_{m}\right) \leqslant \int_{D} Q(z) \cdot \eta_{m}^{2}(z) d m(z),
$$

i.e., $M\left(f \Gamma_{m}\right) \rightarrow 0$ as $m \rightarrow \infty$ in view of (5.1).

The obtained contradiction disproves the assumption that the cluster set $C(P, f)$ consists of more than one point.

Thus, we have the extension $h$ of $f$ to $\bar{D}_{P}$ such that $C\left(E_{D}, f\right) \subseteq \partial D^{\prime}$. In fact, $C\left(E_{D}, f\right)=\partial D^{\prime}$. Indeed, if $\zeta_{0} \in D^{\prime}$, then there is a sequence $\zeta_{n}$ in $D^{\prime}$ being convergent to $\zeta_{0}$. We may assume with no loss of generality that $f^{-1}\left(\zeta_{n}\right) \rightarrow P_{0} \in E_{D}$, because $\bar{D}_{P}$ is compact, see Remark 1.1. Hence, $\zeta_{0} \in C\left(P_{0}, f\right)$. 
Finally, let us show that the extended mapping $h: \bar{D}_{P} \rightarrow \overline{D^{\prime}}$ is continuous. Indeed, let $P_{n} \rightarrow P_{0}$ in $\bar{D}_{P}$. If $P_{0} \in D$, then the statement is obvious. If $P_{0} \in E_{D}$, then, by the last item, we are able to choose $P_{n}^{*} \in D$ such that $\rho\left(P_{n}, P_{n}^{*}\right)<1 / n$, where $\rho$ is one of the metrics in Remark 1.1 and $\left|h\left(P_{n}\right)-h\left(P_{n}^{*}\right)\right|<1 / n$. Note that, by the first part of the proof, $h\left(P_{n}^{*}\right) \rightarrow h\left(P_{0}\right)$ because $P_{n}^{*} \rightarrow P_{0}$. Consequently, $h\left(P_{n}\right) \rightarrow h\left(P_{0}\right)$, too.

Theorem 5.1. Let $D$ and $D^{\prime}$ be bounded finitely connected domains in $\mathbb{C}$ and let $f: D \rightarrow D^{\prime}$ be a ring $Q_{z_{0}}$-homeomorphism at every point $z_{0} \in \partial D$. If

$$
\int_{0}^{\varepsilon\left(z_{0}\right)} \frac{d r}{\left\|Q_{z_{0}}\right\|(r)}=\infty \quad \forall z_{0} \in \partial D,
$$

where $0<\varepsilon\left(z_{0}\right)<d\left(z_{0}\right):=\sup _{z \in D}\left|z-z_{0}\right|$ and

$$
\left\|Q_{z_{0}}\right\|(r):=\int_{D \cap S\left(z_{0}, r\right)} Q_{z_{0}} d s
$$

then $f$ can be extended to a continuous mapping of $\bar{D}_{P}$ onto ${\overline{D^{\prime}}}_{P}$.

Proof. Indeed, condition (5.3) implies that

$$
\int_{0}^{\varepsilon_{0}} \frac{d r}{\left\|Q_{z_{0}}\right\|(r)}=\infty \quad \forall z_{0} \in \partial D \quad \forall \varepsilon_{0} \in\left(0, \varepsilon\left(z_{0}\right)\right)
$$

because the left-hand side in (4.3) is not equal to zero, see Theorem 5.2 in [30], and hence, by Lemma 4.1,

$$
\int_{\varepsilon_{0}}^{\varepsilon\left(z_{0}\right)} \frac{d r}{\left\|Q_{z_{0}}\right\|(r)}<\infty
$$

On the other hand, for the functions

$$
\psi_{z_{0}, \varepsilon_{0}}(t):= \begin{cases}1 /\left\|Q_{z_{0}}\right\|(t), & t \in\left(0, \varepsilon_{0}\right), \\ 0, & t \in\left[\varepsilon_{0}, \infty\right),\end{cases}
$$

we have, by the Fubini theorem, that

$$
\int_{D\left(z_{0}, \varepsilon, \varepsilon_{0}\right)} Q_{z_{0}}(z) \cdot \psi_{z_{0}, \varepsilon_{0}}^{2}\left(\left|z-z_{0}\right|\right) d m(z)=\int_{\varepsilon}^{\varepsilon_{0}} \frac{d r}{\left\|Q_{z_{0}}\right\|(r)}
$$


and, consequently, condition (5.1) holds by (5.5) for all $z_{0} \in \partial D$ and $\varepsilon_{0} \in\left(0, \varepsilon\left(z_{0}\right)\right)$.

Here we have used the standard conventions in the integral theory that $a / \infty=0$ for $a \neq \infty$ and $0 \cdot \infty=0$, see, e.g., Section I.3 in [48].

Thus, Theorem 5.1 follows immediately from Lemma 5.1.

\section{The extension of the inverse mappings to the boundary}

The proof of the extension of the inverse mappings for a ring $Q$-homeomorphism by prime ends in the plane is based on the following lemma on the cluster sets.

Lemma 6.1. Let $D$ and $D^{\prime}$ be bounded finitely connected domains in $\mathbb{C}$, and let $P_{0}$ and $P_{*}$ be prime ends of $D, P_{*} \neq P_{0}$. Denote, by $\sigma_{m}$, $m=1,2, \ldots$, a chain of cross-cuts in $P_{0}$ from Lemma 1.1 lying on circles $S\left(z_{0}, r_{m}\right), z_{0} \in \partial D$, with associated domains $d_{m}$. Suppose that $Q$ is integrable over $D \cap S\left(z_{0}, r\right)$ for a set $E$ of numbers $r \in(0, \delta)$ of a positive linear measure, where $\delta=r_{m_{0}}$ and $m_{0}$ is such that the domain $d_{m_{0}}$ does not contain sequences of points converging to $P_{*}$. If $f: D \rightarrow D^{\prime}$ is a ring $Q$-homeomorphism at the point $z_{0}$ and $\partial D^{\prime}$ is weakly flat, then

$$
C\left(P_{0}, f\right) \cap C\left(P_{*}, f\right)=\varnothing .
$$

Note that, in view of the metrizability of the completion $\bar{D}_{P}$ of the domain $D$ with prime ends, see Remark 1.1, the number $m_{0}$ in Lemma 6.1 always exists.

Proof. Let us choose $\varepsilon \in(0, \delta)$ such that $E_{0}:=\{r \in E: r \in(\varepsilon, \delta)\}$ has a positive linear measure. Such a choice is possible in view of the subadditivity of the linear measure and the exhaustion $E=\cup E_{n}$, where $E_{n}=\{r \in E: r \in(1 / n, \delta)\}, n=1,2, \ldots$. Note that, by Lemma 4.1 for $S_{1}=S\left(z_{0}, \varepsilon\right)$ and $S_{2}=S\left(z_{0}, \delta\right)$,

$$
M\left(\Delta\left(f S_{1}, f S_{2} ; f D\right)\right)<\infty .
$$

Let us assume that $C_{0} \cap C_{*} \neq \varnothing$, where $C_{0}=C\left(P_{0}, f\right)$ and $C_{*}=$ $C\left(P_{*}, f\right)$. By construction, there is $m_{1}>m_{0}$ such that $\sigma_{m_{1}}$ lies on the circle $S\left(z_{0}, r_{m_{1}}\right)$ with $r_{m_{1}}<\varepsilon$. Let $d_{0}=d_{m_{1}}$ and $d_{*} \subseteq D \backslash d_{m_{0}}$ be a domain associated with a chain of cross-cuts in the prime end $P_{*}$. Let $\zeta_{0} \in C_{1} \cap C_{2}$. Choose $\rho_{0}>0$ such that $S\left(\zeta_{0}, \rho_{0}\right) \cap f\left(d_{0}\right) \neq \varnothing$ and $S\left(\zeta_{0}, \rho_{0}\right) \cap f\left(d_{*}\right) \neq \varnothing$.

Set $\Gamma=\Delta\left(\overline{d_{0}}, \overline{d_{*}} ; D\right)$. Correspondingly to $(6.2)$, by the principle of minorization,

$$
M(f(\Gamma))<\infty
$$


Let $M_{0}>M(f(\Gamma))$ be a finite number. By the condition of the lemma, $\partial D^{\prime}$ is weakly flat, and, hence, there is $\rho_{*} \in\left(0, \rho_{0}\right)$ such that

$$
M\left(\Delta\left(E, F ; D^{\prime}\right)\right) \geqslant M_{0}
$$

for all continua $E$ and $F$ in $D^{\prime}$ intersecting the circles $S\left(\zeta_{0}, \rho_{0}\right)$ and $S\left(\zeta_{0}, \rho_{*}\right)$. However, these circles can be joined by continuous curves $c_{1}$ and $c_{2}$ in the domains $f\left(d_{0}\right)$ and $f\left(d_{*}\right)$, correspondingly, and, in particular, for these curves,

$$
M_{0} \leqslant M\left(\Delta\left(c_{1}, c_{2} ; D^{\prime}\right)\right) \leqslant M(f(\Gamma)) .
$$

The obtained contradiction disproves the assumption that $C_{0} \cap C_{*} \neq$ $\varnothing$.

Theorem 6.1. Let $D$ and $D^{\prime}$ be bounded finitely connected domains in $\mathbb{C}$ and $f: D \rightarrow D^{\prime}$ be a $Q_{z_{0}}$-homeomorphism at every point $z_{0} \in \partial D$ with $Q_{z_{0}} \in L^{1}\left(D \cap U_{z_{0}}\right)$ for a neighborhood $U_{z_{0}}$ of $z_{0}$. Then $f^{-1}$ can be extended to a continuous mapping of ${\overline{D^{\prime}}}_{P}$ onto $\bar{D}_{P}$.

Proof. By Remark 1.1, we may assume with no loss of generality that $D^{\prime}$ is a circular domain, $\overline{D^{\prime}}{ }_{P}=\overline{D^{\prime}} ; C\left(\zeta_{0}, f^{-1}\right) \neq \varnothing$ for every $\zeta_{0} \in \partial D^{\prime}$ because $\bar{D}_{P}$ is metrizable and compact. Moreover, $C\left(\zeta_{0}, f^{-1}\right) \cap D=\varnothing$, see, e.g., Proposition 2.5 in [36] or Proposition 13.5 in [26].

Let us assume that there exist at least two different prime ends $P_{1}$ and $P_{2}$ in $C\left(\zeta_{0}, f^{-1}\right)$. Then $\zeta_{0} \in C\left(P_{1}, f\right) \cap C\left(P_{2}, f\right)$. Let $z_{1} \in \partial D$ be a point corresponding to $P_{1}$ from Lemma 1.1. Note that

$$
E=\left\{r \in(0, \delta):\left.Q_{z_{1}}\right|_{D \cap S\left(z_{1}, r\right)} \in L^{1}\left(D \cap S\left(z_{1}, r\right)\right)\right\}
$$

has a positive linear measure for every $\delta>0$ by the Fubini theorem, see, e.g., [48], because $Q_{z_{1}} \in L^{1}\left(D \cap U_{z_{1}}\right)$. The obtained contradiction with Lemma 6.1 shows that $C\left(\zeta_{0}, f^{-1}\right)$ contains only one prime end of $D$.

Thus, we have the extension $g$ of $f^{-1}$ to $\overline{D^{\prime}}$ such that $C\left(\partial D^{\prime}, f^{-1}\right) \subseteq$ $\bar{D}_{P} \backslash D$. In fact, $C\left(\partial D^{\prime}, f^{-1}\right)=\bar{D}_{P} \backslash D$. Indeed, if $P_{0}$ is a prime end of $D$, then there is a sequence $z_{n}$ in $D$ being convergent to $P_{0}$. We may assume without loss of generality that $z_{n} \rightarrow z_{0} \in \partial D$ and $f\left(z_{n}\right) \rightarrow \zeta_{0} \in \partial D^{\prime}$ because $\bar{D}$ and $\overline{D^{\prime}}$ are compact. Hence, $P_{0} \in C\left(\zeta_{0}, f^{-1}\right)$.

Finally, let us show that the extended mapping $g: \bar{D}^{\prime} \rightarrow \bar{D}_{P}$ is continuous. Indeed, let $\zeta_{n} \rightarrow \zeta_{0}$ in $\overline{D^{\prime}}$. If $\zeta_{0} \in D^{\prime}$, then the statement is obvious. If $\zeta_{0} \in \partial D^{\prime}$, then we take $\zeta_{n}^{*} \in D^{\prime}$ such that $\left|\zeta_{n}-\zeta_{n}^{*}\right|<1 / n$ and $\rho\left(g\left(\zeta_{n}\right), g\left(\zeta_{n}^{*}\right)\right)<1 / n$, where $\rho$ is one of the metrics in Remark 1.1. Note that, by construction, $g\left(\zeta_{n}^{*}\right) \rightarrow g\left(\zeta_{0}\right)$ because $\zeta_{n}^{*} \rightarrow \zeta_{0}$. Consequently, $g\left(\zeta_{n}\right) \rightarrow g\left(\zeta_{0}\right)$, too. 
Theorem 6.2. Let $D$ and $D^{\prime}$ be bounded finitely connected domains in $\mathbb{C}$ and let $f: D \rightarrow D^{\prime}$ be a $Q_{z_{0}}$-homeomorphism at every point $z_{0} \in \partial D$ with the condition

$$
\int_{0}^{\varepsilon\left(z_{0}\right)} \frac{d r}{\left\|Q_{z_{0}}\right\|(r)}=\infty
$$

where $0<\varepsilon\left(z_{0}\right)<d\left(z_{0}\right)=\sup _{z \in D}\left|z-z_{0}\right|$ and

$$
\left\|Q_{z_{0}}\right\|(r)=\int_{D \cap S\left(z_{0}, r\right)} Q_{z_{0}} d s
$$

Then $f^{-1}$ can be extended to a continuous mapping of ${\overline{D^{\prime}}}_{P}$ onto $\bar{D}_{P}$.

Proof. Indeed, condition (6.6) implies that

$$
\int_{0}^{\delta} \frac{d r}{\left\|Q_{z_{0}}\right\|(r)}=\infty \quad \forall z_{0} \in \partial D \quad \forall \delta \in\left(0, \varepsilon\left(z_{0}\right)\right)
$$

because the left-hand side in (4.3) is not equal to zero, see Theorem 5.2 in [30], and hence, by Lemma 4.1,

$$
\int_{\delta}^{\varepsilon\left(z_{0}\right)} \frac{d r}{\left\|Q_{z_{0}}\right\|(r)}<\infty
$$

Thus, the set

$$
E=\left\{r \in(0, \delta):\left.Q_{z_{0}}\right|_{D \cap S\left(z_{0}, r\right)} \in L^{1}\left(D \cap S\left(z_{0}, r\right)\right)\right\}
$$

has a positive linear measure for all $z_{0} \in \partial D$ and all $\delta \in\left(0, \varepsilon\left(z_{0}\right)\right)$. The rest of arguments is perfectly similar to one in the proof of Theorem 6.1.

\section{The homeomorphic extension of ring $Q$-homeomorphisms}

Combining Theorems 5.1 and 6.2, we arrive at Theorem 7.1, that is a counterpart of the classic Carathéodory theorem on conformal mappings of simply connected domains. 
Theorem 7.1. Let $D$ and $D^{\prime}$ be bounded finitely connected domains in $\mathbb{C}$ and let $f: D \rightarrow D^{\prime}$ be a ring $Q_{z_{0}}$-homeomorphism at every point $z_{0} \in \partial D$. If

$$
\int_{0}^{\varepsilon\left(z_{0}\right)} \frac{d r}{\left\|Q_{z_{0}}\right\|(r)}=\infty \quad \forall z_{0} \in \partial D
$$

where $0<\varepsilon\left(z_{0}\right)<d\left(z_{0}\right):=\sup _{z \in D}\left|z-z_{0}\right|$ and

$$
\left\|Q_{z_{0}}\right\|(r):=\int_{D \cap S\left(z_{0}, r\right)} Q_{z_{0}} d s
$$

then $f$ can be extended to a homeomorphism of $\bar{D}_{P}$ onto ${\overline{D^{\prime}}}_{P}$.

Corollary 7.1. In particular, the conclusion of Theorem 7.1 holds if

$$
q_{z_{0}}(r)=O\left(\log \frac{1}{r}\right) \quad \forall z_{0} \in \partial D
$$

as $r \rightarrow 0$, where $q_{z_{0}}(r)$ is the average of $Q_{z_{0}}$ over the circle $\left|z-z_{0}\right|=r$.

Using Lemma 2.2 in [38], see also Lemma 7.4 in [26], and Theorem 7.1, we get the following statement, which is a source of new criteria for the homeomorphic extendability.

Lemma 7.1. Let $D$ and $D^{\prime}$ be bounded finitely connected domains in $\mathbb{C}$ and let $f: D \rightarrow D^{\prime}$ be a ring $Q_{z_{0}}$-homeomorphism at every point $z_{0} \in \partial D$, where $Q_{z_{0}}$ is integrable in a neighborhood of $z_{0}$. Suppose that

$$
\int_{\left(z_{0}, \varepsilon, \varepsilon_{0}\right)} Q_{z_{0}}(z) \cdot \psi_{z_{0}, \varepsilon, \varepsilon_{0}}^{2}\left(\left|z-z_{0}\right|\right) d m(z)=o\left(I_{z_{0}, \varepsilon_{0}}^{2}(\varepsilon)\right) \quad \text { as } \varepsilon \rightarrow 0 \forall z_{0} \in \partial D
$$

where $D\left(z_{0}, \varepsilon, \varepsilon_{0}\right)=\left\{z \in D: \varepsilon<\left|z-z_{0}\right|<\varepsilon_{0}\right\}$ for every small enough $0<\varepsilon_{0}<d\left(z_{0}\right)=\sup _{z \in D}\left|z-z_{0}\right|$ and where $\psi_{z_{0}, \varepsilon, \varepsilon_{0}}(t):(0, \infty) \rightarrow[0, \infty]$, $\varepsilon \in\left(0, \varepsilon_{0}\right)$, is a family of (Lebesgue) measurable functions such that

$$
0<I_{z_{0}, \varepsilon_{0}}(\varepsilon):=\int_{\varepsilon}^{\varepsilon_{0}} \psi_{z_{0}, \varepsilon, \varepsilon_{0}}(t) d t<\infty \quad \forall \varepsilon \in\left(0, \varepsilon_{0}\right) .
$$

Then $f$ can be extended to a homeomorphism of $\bar{D}_{P}$ onto ${\overline{D^{\prime}}}_{P}$.

Remark 7.1. In fact, instead of the integrability of $Q_{z_{0}}$ in a neighborhood of $z_{0}$, it is sufficient to request that $Q_{z_{0}}$ be integrable over $D \cap S\left(z_{0}, r\right)$ for a.e. $r \in\left(0, \varepsilon_{0}\right)$. 
Note that Theorem 7.1 can be deduced also from Lemma 7.1, as it follows from the proof of Theorem 5.1. Thus, Theorem 7.1 is equivalent to Lemma 7.1 under the given conditions.

Finally, note that (7.4) holds, in particular, if

$$
\int_{D\left(z_{0}, \varepsilon_{0}\right)} Q_{z_{0}}(z) \cdot \psi^{2}\left(\left|z-z_{0}\right|\right) d m(z)<\infty \quad \forall z_{0} \in \partial D
$$

where $D\left(z_{0}, \varepsilon_{0}\right)=\left\{z \in D:\left|z-z_{0}\right|<\varepsilon_{0}\right\}$ and where $\psi(t):(0, \infty) \rightarrow$ $[0, \infty]$ is a locally integrable function such that $I_{z_{0}, \varepsilon_{0}}(\varepsilon) \rightarrow \infty$ as $\varepsilon \rightarrow 0$. In other words, for the extendability of $f$ to a homeomorphism of $\bar{D}_{P}$ onto $\overline{D^{\prime}}{ }_{P}$, it suffices for the integrals in (7.5) to be convergent for some nonnegative function $\psi(t)$ that is locally integrable on $(0, \infty)$ but has a non-integrable singularity at zero.

Choosing $\psi(t):=\frac{1}{t \log 1 / t}$ in Lemma 7.1 and applying Lemma 3.1, we obtain the following proposition.

Theorem 7.2. Let $D$ and $D^{\prime}$ be bounded finitely connected domains in $\mathbb{C}$ and let $f: D \rightarrow D^{\prime}$ be a ring $Q_{z_{0}}$-homeomorphism at every point $z_{0} \in \partial D$, where $Q_{z_{0}}$ has finite mean oscillation at $z_{0}$. Then $f$ can be extended to a homeomorphism of $\bar{D}_{P}$ onto ${\overline{D^{\prime}}}_{P}$.

Corollary 7.2. In particular, the conclusion of Theorem 7.2 holds if

$$
\varlimsup_{\varepsilon \rightarrow 0} \int_{B\left(z_{0}, \varepsilon\right)} Q_{z_{0}}(z) d m(z)<\infty \quad \forall z_{0} \in \partial D
$$

Corollary 7.3. The conclusion of Theorem 7.2 holds if every point $z_{0} \in$ $\partial D$ is a Lebesgue point of the function $Q_{z_{0}}$.

The next statement also follows from Lemma 7.1 under the choice $\psi(t)=1 / t$.

Theorem 7.3. Let $D$ and $D^{\prime}$ be bounded finitely connected domains in $\mathbb{C}$ and let $f: D \rightarrow D^{\prime}$ be a ring $Q_{z_{0}}$-homeomorphism at every point $z_{0} \in \partial D$. If, for some $\varepsilon_{0}=\varepsilon\left(z_{0}\right)>0$,

$\int_{\varepsilon<\left|z-z_{0}\right|<\varepsilon_{0}} Q_{z_{0}}(z) \frac{d m(z)}{\left|z-z_{0}\right|^{2}}=o\left(\left[\log \frac{1}{\varepsilon}\right]^{2}\right) \quad$ as $\varepsilon \rightarrow 0 \quad \forall z_{0} \in \partial D$,

then $f$ can be extended to a homeomorphism of $\bar{D}_{P}$ onto ${\overline{D^{\prime}}}_{P}$. 
Remark 7.2. Choosing the function $\psi(t)=1 /(t \log 1 / t)$ instead of $\psi(t)$ $=1 / t$ in Lemma 7.1, (7.7) can be replaced by the more weak condition

$$
\int_{\varepsilon<\left|z-z_{0}\right|<\varepsilon_{0}} \frac{Q_{z_{0}}(z) d m(z)}{\left(\left|z-z_{0}\right| \log \frac{1}{\left|z-z_{0}\right|}\right)^{2}}=o\left(\left[\log \log \frac{1}{\varepsilon}\right]^{2}\right)
$$

and (7.3) by the condition in terms of iterated logarithms

$$
q_{z_{0}}(r)=o\left(\log \frac{1}{r} \log \log \frac{1}{r}\right)
$$

Of course, we could give here the whole scale of the corresponding condition of logarithmic type, using suitable functions $\psi(t)$.

Theorem 7.1 has a lot of other fine consequences, for instance:

Theorem 7.4. Let $D$ and $D^{\prime}$ be bounded finitely connected domains in $\mathbb{C}$ and let $f: D \rightarrow D^{\prime}$ be a ring $Q_{z_{0}}$-homeomorphism at every point $z_{0} \in \partial D$ and

$$
\int_{D \cap B\left(z_{0}, \varepsilon_{0}\right)} \Phi_{z_{0}}\left(Q_{z_{0}}(z)\right) d m(z)<\infty \quad \forall z_{0} \in \partial D
$$

for $\varepsilon_{0}=\varepsilon\left(z_{0}\right)>0$ and a nondecreasing convex function $\Phi_{z_{0}}:[0, \infty) \rightarrow$ $[0, \infty)$ with

$$
\int_{\delta\left(z_{0}\right)}^{\infty} \frac{d \tau}{\tau \Phi_{z_{0}}^{-1}(\tau)}=\infty
$$

for $\delta\left(z_{0}\right)>\Phi_{z_{0}}(0)$. Then $f$ is extended to a homeomorphism of $\bar{D}_{P}$ onto $\bar{D}_{P}^{\prime}$.

Indeed, by Theorem 3.1 and Corollary 3.2 in [46], (7.10) and (7.11) imply (7.1) and, thus, Theorem 7.4 is a direct consequence of Theorem 7.1.

Corollary 7.4. In particular, the conclusion of Theorem 7.4 holds if

$$
\int_{D \cap B\left(z_{0}, \varepsilon_{0}\right)} e^{\alpha_{0} Q_{z_{0}}(z)} d m(z)<\infty \quad \forall z_{0} \in \partial D
$$

for some $\varepsilon_{0}=\varepsilon\left(z_{0}\right)>0$ and $\alpha_{0}=\alpha\left(z_{0}\right)>0$. 
Remark 7.3. By Theorem 2.1 in [46], see also Proposition 2.3 in [39], (7.11) is equivalent to each of the conditions from the following series:

$$
\begin{gathered}
\int_{\delta\left(z_{0}\right)}^{\infty} H_{z_{0}}^{\prime}(t) \frac{d t}{t}=\infty, \quad \delta\left(z_{0}\right)>0, \\
\int_{\delta\left(z_{0}\right)}^{\infty} \frac{d H_{z_{0}}(t)}{t}=\infty, \quad \delta\left(z_{0}\right)>0, \\
\int_{\delta\left(z_{0}\right)}^{\infty} H_{z_{0}}(t) \frac{d t}{t^{2}}=\infty, \quad \delta\left(z_{0}\right)>0, \\
\int_{0\left(z_{0}\right)}^{\infty} H_{z_{0}}\left(\frac{1}{t}\right) d t=\infty, \quad \Delta\left(z_{0}\right)>0, \\
\int_{\delta_{*}\left(z_{0}\right)}^{\infty} \frac{d \eta}{H_{z_{0}}^{-1}(\eta)}=\infty, \quad \delta_{*}\left(z_{0}\right)>H_{z_{0}}(0),
\end{gathered}
$$

where

$$
H_{z_{0}}(t)=\log \Phi_{z_{0}}(t) .
$$

Here the integral in (7.14) is understood as the Lebesgue-Stieltjes integral and the integrals in (7.13) and (7.15)-(7.17) as the ordinary Lebesgue integrals.

It is necessary to give one more explanation. From the right-hand sides in conditions (7.13)-(7.17), we have in mind $+\infty$. If $\Phi_{z_{0}}(t)=0$ for $t \in\left[0, t_{*}\left(z_{0}\right)\right]$, then $H_{z_{0}}(t)=-\infty$ for $t \in\left[0, t_{*}\left(z_{0}\right)\right]$ and we complete the definition $H_{z_{0}}^{\prime}(t)=0$ for $t \in\left[0, t_{*}\left(z_{0}\right)\right]$. Note that conditions $(7.14)$ and (7.15) exclude that $t_{*}\left(z_{0}\right)$ belongs to the interval of integrability because, in the contrary case, the left-hand sides in (7.14) and (7.15) are either equal to $-\infty$ or indeterminate. Hence, we may assume in (7.13)-(7.16) that $\delta\left(z_{0}\right)>t_{0}$, correspondingly, $\Delta\left(z_{0}\right)<1 / t\left(z_{0}\right)$, where $t\left(z_{0}\right):=\sup _{\Phi_{z_{0}}(t)=0} t$, set $t\left(z_{0}\right)=0$ if $\Phi_{z_{0}}(0)>0$.

The most interesting is condition (7.15) that can be written in the form:

$$
\int_{\delta\left(z_{0}\right)}^{\infty} \log \Phi_{z_{0}}(t) \frac{d t}{t^{2}}=\infty .
$$


Note also that, under every homeomorphism $f$ between domains $D$ and $D^{\prime}$ in $\overline{\mathbb{C}}$, there is a natural one-to-one correspondence between components of their boundaries $\partial D$ and $\partial D^{\prime}$, see, e.g., Lemma 5.3 in [14] or Lemma 6.5 in [26]. Thus, if a bounded domain $D$ in $\mathbb{C}$ is finitely connected and $D^{\prime}$ is bounded, then $D^{\prime}$ is finitely connected, too.

Finally, note that if a domain $D$ in $\mathbb{C}$ is locally connected on its boundary, then there is a natural one-to-one correspondence between prime ends of $D$ and boundary points of $D$. Thus, if $D$ and $D^{\prime}$ are, in addition, locally connected on their boundaries in the theorems of Section 7 , then $f$ is extended to a homeomorphism of $\bar{D}$ onto $\overline{D^{\prime}}$. We obtained earlier similar results, when $\partial D^{\prime}$ is weakly flat, which is a more strong condition than that of local connectivity of $D^{\prime}$ on its boundary, see, e.g., [17, 18].

As known, every Jordan domain $D$ in $\mathbb{C}$ is locally connected on its boundary, see, e.g., [53, p. 66]. It is easy to see that the latter implies that every bounded finitely connected domain $D$ in $\mathbb{C}$ whose boundary consists of mutually disjoint Jordan curves and isolated points is also locally connected on its boundary.

Conversely, every bounded finitely connected domain $D$ in $\mathbb{C}$ that is locally connected on its boundary has a boundary consisting of mutually disjoint Jordan curves and isolated points. Indeed, every such a domain $D$ can be mapped by a conformal mapping $f$ onto the so-called circular domain $D_{*}$ bounded by a finite collection of mutually disjoint circles and isolated points, see, e.g., Theorem V.6.2 in [9], that is extendable to a homeomorphism of $\bar{D}$ onto $\overline{D_{*}}$, see Remark 1.1.

\section{The boundary behavior of homeomorphic solutions}

This section is devoted to the study of the boundary behavior of solutions to the Beltrami equations. Combining Theorem 4.1 with the corresponding results in Sections 6 and 7, we obtain the statements given below.

Theorem 8.1. Let $D$ and $D^{\prime}$ be bounded finitely connected domains in $\mathbb{C}$ and let $f: D \rightarrow D^{\prime}$ be a homeomorphic $W_{\mathrm{loc}}^{1,1}$ solution of (1.1) with $K_{\mu}^{T}\left(\cdot, z_{0}\right) \in L^{1}\left(D \cap B\left(z_{0}, \varepsilon_{0}\right)\right)$ for every $z_{0} \in \partial D$. Then $f^{-1}$ is extended to a continuous mapping of ${\overline{D^{\prime}}}_{P}$ onto $\bar{D}_{P}$.

Note that any degree of integrability of $K_{\mu}$ does not guarantee a continuous extendability of the direct mapping $f$ to the boundary, see, e.g., an example in the proof of Proposition 6.3 in [26]. Conditions for the continuous extendability have perfectly another nature. 
Theorem 8.2. Let $D$ and $D^{\prime}$ be bounded finitely connected domains in $\mathbb{C}$ and let $f: D \rightarrow D^{\prime}$ be a homeomorphic $W_{\mathrm{loc}}^{1,1}$ solution of the Beltrami equation (1.1) with the condition

$$
\int_{0}^{\varepsilon_{0}} \frac{d r}{\left\|K_{\mu}^{T}\right\|\left(z_{0}, r\right)}=\infty \quad \forall z_{0} \in \partial D
$$

where $0<\varepsilon_{0}=\varepsilon\left(z_{0}\right)<d\left(z_{0}\right):=\sup _{z \in D}\left|z-z_{0}\right|$ and

$$
\left\|K_{\mu}^{T}\right\|\left(z_{0}, r\right)=\int_{S\left(z_{0}, r\right)} K_{\mu}^{T}\left(z, z_{0}\right) d s .
$$

Then $f$ can be extended to a homeomorphism of $\bar{D}_{P}$ onto ${\overline{D^{\prime}}}_{P}$.

Here and later on, we set that $K_{\mu}^{T}$ is equal to zero outside of the domain $D$.

Corollary 8.1. In particular, the conclusion of Theorem 8.2 holds if

$$
k_{z_{0}}^{T}(r)=O\left(\log \frac{1}{r}\right) \quad \forall z_{0} \in \partial D
$$

as $r \rightarrow 0$ where $k_{z_{0}}^{T}(r)$ is the average of $K_{\mu}^{T}\left(z, z_{0}\right)$ over the circle $\left|z-z_{0}\right|=$ $r$.

Lemma 8.1. Let $D$ and $D^{\prime}$ be bounded finitely connected domains in $\mathbb{C}$ and let $f: D \rightarrow D^{\prime}$ be a homeomorphic $W_{\mathrm{loc}}^{1,1}$ solution of the Beltrami equation (1.1) with $K_{\mu} \in L^{1}(D)$ and

$$
\int_{\varepsilon<\left|z-z_{0}\right|<\varepsilon_{0}} K_{\mu}^{T}\left(z, z_{0}\right) \cdot \psi_{z_{0}, \varepsilon}^{2}\left(\left|z-z_{0}\right|\right) d m(z)=o\left(I_{z_{0}}^{2}(\varepsilon)\right) \quad \forall z_{0} \in \partial D
$$

as $\varepsilon \rightarrow 0$, where $0<\varepsilon_{0}<\sup _{z \in D}\left|z-z_{0}\right|$ and $\psi_{z_{0}, \varepsilon}(t):(0, \infty) \rightarrow[0, \infty]$, $\varepsilon \in\left(0, \varepsilon_{0}\right)$, is a two-parametric family of measurable functions such that

$$
0<I_{z_{0}}(\varepsilon):=\int_{\varepsilon}^{\varepsilon_{0}} \psi_{z_{0}, \varepsilon}(t) d t<\infty \quad \forall \varepsilon \in\left(0, \varepsilon_{0}\right) .
$$

Then $f$ can be extended to a homeomorphism of $\bar{D}_{P}$ onto ${\overline{D^{\prime}}}_{P}$.

Theorem 8.3. Let $D$ and $D^{\prime}$ be bounded finitely connected domains in $\mathbb{C}$ and let $f: D \rightarrow D^{\prime}$ be a homeomorphic $W_{\mathrm{loc}}^{1,1}$ solution of the Beltrami equation (1.1) with $K_{\mu}^{T}\left(z, z_{0}\right)$ of finite mean oscillation at every point $z_{0} \in \partial D$. Then $f$ can be extended to a homeomorphism of $\bar{D}_{P}$ onto ${\overline{D^{\prime}}}_{P}$. 
Theorem 8.3 remains valid also if the function $K_{\mu}^{T}\left(z, z_{0}\right)$ has a dominant of finite mean oscillation in a neighborhood of every point $z_{0} \in \partial D$.

Corollary 8.2. In particular, the conclusion of Theorem 8.3 holds if

$$
\varlimsup_{\varepsilon \rightarrow 0} f_{B\left(z_{0}, \varepsilon\right)} K_{\mu}^{T}\left(z, z_{0}\right) d m(z)<\infty \quad \forall z_{0} \in \partial D .
$$

Theorem 8.4. Let $D$ and $D^{\prime}$ be bounded finitely connected domains in $\mathbb{C}$ and let $f: D \rightarrow D^{\prime}$ be a homeomorphic $W_{\mathrm{loc}}^{1,1}$ solution of the Beltrami equation (1.1) with the condition

$$
\int_{\varepsilon<\left|z-z_{0}\right|<\varepsilon_{0}} K_{\mu}^{T}\left(z, z_{0}\right) \frac{d m(z)}{\left|z-z_{0}\right|^{2}}=o\left(\left[\log \frac{1}{\varepsilon}\right]^{2}\right) \quad \forall z_{0} \in \partial D .
$$

Then $f$ can be extended to a homeomorphism of $\bar{D}_{P}$ onto ${\overline{D^{\prime}}}_{P}$.

Remark 8.1. Condition (8.6) can be replaced by the weaker condition

$$
\int_{\varepsilon<\left|z-z_{0}\right|<\varepsilon_{0}} \frac{K_{\mu}^{T}\left(z, z_{0}\right) d m(z)}{\left(\left|z-z_{0}\right| \log \frac{1}{\left|z-z_{0}\right|}\right)^{2}}=o\left(\left[\log \log \frac{1}{\varepsilon}\right]^{2}\right) \quad \forall z_{0} \in \partial D .
$$

In general, here we are able to give a number of other conditions of logarithmic type. In particular, condition (8.3) can be replaced, due to Theorem 8.2 , by the weaker condition

$$
k_{z_{0}}^{T}(r)=O\left(\log \frac{1}{r} \log \log \frac{1}{r}\right) .
$$

Finally, we complete the series of criteria with the following integral condition.

Theorem 8.5. Let $D$ and $D^{\prime}$ be bounded finitely connected domains in $\mathbb{C}$ and let $f: D \rightarrow D^{\prime}$ be a homeomorphic $W_{\mathrm{loc}}^{1,1}$ solution of the Beltrami equation (1.1) with the condition

$$
\int_{D \cap B\left(z_{0}, \varepsilon_{0}\right)} \Phi_{z_{0}}\left(K_{\mu}^{T}\left(z, z_{0}\right)\right) d m(z)<\infty \quad \forall z_{0} \in \partial D
$$

for $\varepsilon_{0}=\varepsilon\left(z_{0}\right)>0$ and a nondecreasing convex function $\Phi_{z_{0}}:[0, \infty) \rightarrow$ $[0, \infty)$ with

$$
\int_{\delta_{0}}^{\infty} \frac{d \tau}{\tau \Phi_{z_{0}}^{-1}(\tau)}=\infty
$$


for $\delta_{0}=\delta\left(z_{0}\right)>\Phi_{z_{0}}(0)$. Then $f$ is extended to a homeomorphism of $\bar{D}_{P}$ onto $\overline{D^{\prime}} P$.

Corollary 8.3. In particular, the conclusion of Theorem 8.5 holds if

$$
\int_{D \cap B\left(z_{0}, \varepsilon_{0}\right)} e^{\alpha_{0} K_{\mu}^{T}\left(z, z_{0}\right)} d m(z)<\infty \quad \forall z_{0} \in \partial D
$$

for some $\varepsilon_{0}=\varepsilon\left(z_{0}\right)>0$ and $\alpha_{0}=\alpha\left(z_{0}\right)>0$.

Remark 8.2. Note that condition (8.10) is not only sufficient but also necessary for a continuous extension to the boundary of all direct mappings $f$ with integral restrictions of type (8.9), see, e.g., Theorem 5.1 and Remark 5.1 in [22]. In other words, given $\Phi_{z_{0}}$ which does not satisfy (8.10), one can find a homeomorphic $W_{\text {loc }}^{1,1}$ solution of (1.1) with condition (8.9) that is not extended to a homeomorphism of $\bar{D}_{P}$ onto ${\overline{D^{\prime}}}_{P}$.

Recall also that condition (8.10) is equivalent to each of conditions (7.13)-(7.17).

\section{Regular solutions for the Dirichlet problem}

Recall that a mapping $f: D \rightarrow \mathbb{C}$ is called discrete if the pre-image $f^{-1}(y)$ of every point $y \in \mathbb{C}$ consists of isolated points and open if the image of every open set $U \subseteq D$ is open in $\mathbb{C}$.

Given $\varphi(P) \not \equiv$ const, $P \in E_{D}$, we will say that $f$ is a regular solution of the Dirichlet problem (1.6) for the Beltrami equation (1.1) if $f$ is a continuous discrete open mapping $f: D \rightarrow \mathbb{C}$ of the Sobolev class $W_{\text {loc }}^{1,1}$ with the Jacobian

$$
J_{f}(z)=\left|f_{z}\right|^{2}-\left|f_{\bar{z}}\right|^{2} \neq 0 \quad \text { a.e., }
$$

satisfying (1.1) a.e. and the boundary condition (1.6) for all prime ends of the domain $D$. For $\varphi(P) \equiv c \in \mathbb{R}, P \in E_{D}$, a regular solution of the problem is any constant function $f(z)=c+i c^{\prime}, c^{\prime} \in \mathbb{R}$.

Theorem 9.1. Let $D$ be a bounded simply connected domain in $\mathbb{C}$ and let $\mu: D \rightarrow \mathbb{D}$ be a measurable function with $K_{\mu} \in L_{\text {loc }}^{1}$ and such that

$$
\int_{0}^{\delta\left(z_{0}\right)} \frac{d r}{\left\|K_{\mu}^{T}\right\|\left(z_{0}, r\right)}=\infty \quad \forall z_{0} \in \bar{D}
$$

for some $0<\delta\left(z_{0}\right)<d\left(z_{0}\right)=\sup _{z \in D}\left|z-z_{0}\right|$, where

$$
\left\|K_{\mu}^{T}\right\|\left(z_{0}, r\right):=\int_{S\left(z_{0}, r\right)} K_{\mu}^{T}\left(z, z_{0}\right) d s .
$$


Then the Beltrami equation (1.1) has a regular solution $f$ of the Dirichlet problem (1.6) for every continuous function $\varphi: E_{D} \rightarrow \mathbb{R}$.

Here and later on, we set that $K_{\mu}^{T}$ is equal to zero outside of the domain $D$.

Corollary 9.1. Let $D$ be a bounded simply connected domain in $\mathbb{C}$ and let $\mu: D \rightarrow \mathbb{D}$ be a measurable function such that

$$
k_{z_{0}}^{T}(\varepsilon)=O\left(\log \frac{1}{\varepsilon}\right) \quad \text { as } \varepsilon \rightarrow 0 \quad \forall z_{0} \in \bar{D},
$$

where $k_{z_{0}}^{T}(\varepsilon)$ is the average of the function $K_{\mu}^{T}\left(z, z_{0}\right)$ over the circle $S\left(z_{0}, \varepsilon\right)$.

Then the Beltrami equation (1.1) has a regular solution $f$ of the Dirichlet problem (1.6) for every continuous function $\varphi: E_{D} \rightarrow \mathbb{R}$.

Remark 9.1. In particular, the conclusion of Theorem 9.1 holds if

$$
K_{\mu}^{T}\left(z, z_{0}\right)=O\left(\log \frac{1}{\left|z-z_{0}\right|}\right) \quad \text { as } \quad z \rightarrow z_{0} \quad \forall z_{0} \in \bar{D} .
$$

Proof of Theorem 9.1. Note that $E_{D}$ cannot consist of a single prime end. Indeed, all rays going from a point $z_{0} \in D$ to $\infty$ intersect $\partial D$ because the domain $D$ is bounded, see, e.g., Proposition 2.3 in [36] or Proposition 13.3 in [26]. Thus, $\partial D$ contains more than one point and, by the Riemann theorem, see, e.g., II.2.1 in [9], $D$ can be mapped onto the unit disk $\mathbb{D}$ with a conformal mapping $R$. However, then there is one-toone correspondence between elements of $E_{D}$ and points of the unit circle $\partial \mathbb{D}$ by the Carathéodory theorem, see, e.g., Theorem 9.6 in [5].

Let $F$ be a regular homeomorphic solution of Eq. (1.1) in the class $W_{\text {loc }}^{1,1}$ which exists in view of condition (9.2), see, e.g., Theorem 5.4 in work [41] or Theorem 11.10 in book [26].

Note that the domain $D^{*}=F(D)$ is simply connected in $\overline{\mathbb{C}}$, see, e.g., Lemma 5.3 in [14] or Lemma 6.5 in [26]. Let us assume that $\partial D^{*}$ in $\overline{\mathbb{C}}$ consists of the single point $\infty$. Then $\overline{\mathbb{C}} \backslash D^{*}$ also consists of the single point $\infty$, i.e., $D^{*}=\mathbb{C}$. Indeed, if there is a point $\zeta_{0} \in \mathbb{C}$ in $\overline{\mathbb{C}} \backslash D^{*}$, then, joining it and any point $\zeta_{*} \in D^{*}$ with a segment of a straight line, we find one more point of $\partial D^{*}$ in $\mathbb{C}$, see, e.g., again Proposition 2.3 in [36] or Proposition 13.3 in [26]. Now, let $\mathbb{D}^{*}$ denote the exterior of the unit $\operatorname{disk} \mathbb{D}$ in $\mathbb{C}$ and let $\kappa(\zeta)=1 / \zeta, \kappa(0)=\infty, \kappa(\infty)=0$. Consider the mapping $F_{*}=\kappa \circ F: \widetilde{D} \rightarrow \mathbb{D}_{0}$, where $\widetilde{D}=F^{-1}\left(\mathbb{D}^{*}\right)$ and $\mathbb{D}_{0}=\mathbb{D} \backslash\{0\}$ is the punctured unit disk. It is clear that $F_{*}$ is also a regular homeomorphic solution of the Beltrami equation (1.1) in the class $W_{\text {loc }}^{1,1}$ in the 
bounded two-connected domain $\widetilde{D}$ because the mapping $\kappa$ is conformal. By Theorem 8.2, there is a one-to-one correspondence between elements of $E_{D}$ and 0 . However, it was shown above that $E_{D}$ cannot consists of a single prime end. This contradiction disproves the above assumption that $\partial D^{*}$ consists of a single point in $\overline{\mathbb{C}}$.

Thus, by the Riemann theorem, $D^{*}$ can be mapped onto the unit disk $\mathbb{D}$ with a conformal mapping $R_{*}$. Note that the function $g:=R_{*} \circ F$ is again a regular homeomorphic solution in the Sobolev class $W_{\text {loc }}^{1,1}$ of the Beltrami equation (1.1) which maps $D$ onto $\mathbb{D}$. By Theorem 8.2, the mapping $g$ admits an extension to a homeomorphism $g_{*}: \bar{D}_{P} \rightarrow \overline{\mathbb{D}}$.

We find a regular solution of the initial Dirichlet problem (1.6) in the form $f=h \circ g$, where $h$ is a holomorphic function in $\mathbb{D}$ with the boundary condition

$$
\lim _{z \rightarrow \zeta} \operatorname{Re} h(z)=\varphi\left(g_{*}^{-1}(\zeta)\right) \quad \forall \zeta \in \partial \mathbb{D} .
$$

Note that we have from the right-hand side a continuous function of the variable $\zeta$.

As known, the analytic function $h$ can be reconstructed in $\mathbb{D}$ through its real part on the boundary up to a pure imaginary additive constant with the Schwartz formula, see, e.g., $\S 8$, Chapter III, Part 3 in [13],

$$
h(z)=\frac{1}{2 \pi i} \int_{|\zeta|=1} \varphi \circ g_{*}^{-1}(\zeta) \cdot \frac{\zeta+z}{\zeta-z} \cdot \frac{d \zeta}{\zeta} .
$$

It is easy to see that the function $f=h \circ g$ is a desired regular solution of the Dirichlet problem (1.6) for the Beltrami equation (1.1).

Applying Lemma 2.2 in [38], see also Lemma 7.4 in [26], we obtain the following general lemma immediately from Theorem 9.1.

Lemma 9.1. Let $D$ be a bounded simply connected domain in $\mathbb{C}$ and let $\mu: D \rightarrow \mathbb{D}$ be a measurable function with $K_{\mu} \in L^{1}(D)$. Suppose that, for every $z_{0} \in \bar{D}$ and every small enough $\varepsilon_{0}<d\left(z_{0}\right):=\sup _{z \in D}\left|z-z_{0}\right|$, there is a family of measurable functions $\psi_{z_{0}, \varepsilon, \varepsilon_{0}}:(0, \infty) \rightarrow[0, \infty], \varepsilon \in\left(0, \varepsilon_{0}\right)$ such that

$$
0<I_{z_{0}, \varepsilon_{0}}(\varepsilon):=\int_{\varepsilon}^{\varepsilon_{0}} \psi_{z_{0}, \varepsilon, \varepsilon_{0}}(t) d t<\infty \quad \forall \varepsilon \in\left(0, \varepsilon_{0}\right)
$$

and

$$
\int_{D\left(z_{0}, \varepsilon, \varepsilon_{0}\right)} K_{\mu}^{T}\left(z, z_{0}\right) \cdot \psi_{z_{0}, \varepsilon, \varepsilon_{0}}^{2}\left(\left|z-z_{0}\right|\right) d m(z)=o\left(I_{z_{0}, \varepsilon_{0}}^{2}(\varepsilon)\right) \quad \text { as } \varepsilon \rightarrow 0,
$$


where $D\left(z_{0}, \varepsilon, \varepsilon_{0}\right)=\left\{z \in D: \varepsilon<\left|z-z_{0}\right|<\varepsilon_{0}\right\}$. Then the Beltrami equation (1.1) has a regular solution $f$ of the Dirichlet problem (1.6) for every continuous function $\varphi: E_{D} \rightarrow \mathbb{R}$.

Remark 9.2. In fact, it is sufficient here to request instead of the condition $K_{\mu} \in L^{1}(D)$ only a local integrability of $K_{\mu}$ in the domain $D$ and the condition $\left\|K_{\mu}\right\|\left(z_{0}, r\right) \neq \infty$ for a.e. $r \in\left(0, \varepsilon_{0}\right)$ at all $z_{0} \in \partial D$.

By Lemma 9.1 with the choice $\psi_{z_{0}, \varepsilon}(t) \equiv 1 /\left(t \log \frac{1}{t}\right)$, we obtain the following result, see also Lemma 3.1.

Theorem 9.2. Let $D$ be a bounded simply connected domain in $\mathbb{C}$ and let $\mu: D \rightarrow \mathbb{D}$ be a measurable function with $K_{\mu}^{T} \in L_{\text {loc }}^{1}$ and

$$
K_{\mu}^{T}\left(z, z_{0}\right) \leqslant Q_{z_{0}}(z) \in \operatorname{FMO}\left(z_{0}\right) \quad \forall z_{0} \in \bar{D} .
$$

Then the Beltrami equation (1.1) has a regular solution $f$ of the Dirichlet problem (1.6) for every continuous function $\varphi: E_{D} \rightarrow \mathbb{R}$.

Remark 9.3. In particular, the hypotheses and the conclusion of Theorem 9.2 hold if either $Q_{z_{0}} \in \mathrm{BMO}_{\text {loc }}$ or $Q_{z_{0}} \in \mathrm{W}_{\text {loc }}^{1,2}$ because $W_{\text {loc }}^{1,2} \subset$ $\mathrm{VMO}_{\text {loc }}$, see, e.g., [3].

By Corollary 3.1, we obtain the following statement from Theorem 9.2.

Corollary 9.2. Let $D$ be a bounded simply connected domain in $\mathbb{C}$ and let $\mu: D \rightarrow \mathbb{D}$ be a measurable function with $K_{\mu} \in L_{\text {loc }}^{1}$ such that

$$
\limsup _{\varepsilon \rightarrow 0} f_{B\left(z_{0}, \varepsilon\right)} K_{\mu}^{T}\left(z, z_{0}\right) d m(z)<\infty \quad \forall z_{0} \in \bar{D} .
$$

Then the Beltrami equation (1.1) has a regular solution $f$ of the Dirichlet problem (1.6) for every continuous function $\varphi: E_{D} \rightarrow \mathbb{R}$.

Remark 9.4. In particular, by (1.5), the conclusion of Theorem 9.2 holds if

$$
K_{\mu}(z) \leqslant Q(z) \in \operatorname{BMO}(\bar{D})
$$

The next statement follows from Lemma 9.1 under the choice $\psi(t)=$ $1 / t$, see also Remark 9.2.

Theorem 9.3. Let $D$ be a bounded simply connected domain in $\mathbb{C}$ and let $\mu: D \rightarrow \mathbb{D}$ be a measurable function such that

$$
\int_{\varepsilon<\left|z-z_{0}\right|<\varepsilon_{0}} K_{\mu}^{T}\left(z, z_{0}\right) \frac{d m(z)}{\left|z-z_{0}\right|^{2}}=o\left(\left[\log \frac{1}{\varepsilon}\right]^{2}\right) \quad \forall z_{0} \in \bar{D} .
$$

Then the Beltrami equation (1.1) has a regular solution $f$ of the Dirichlet problem (1.6) for every continuous function $\varphi: E_{D} \rightarrow \mathbb{R}$. 
Remark 9.5. Similarly, choosing $\psi(t)=1 /(t \log 1 / t)$ instead of $\psi(t)=$ $1 / t$ in Lemma 9.1, we obtain that condition (9.10) can be replaced by the condition

$$
\int_{\varepsilon<\left|z-z_{0}\right|<\varepsilon_{0}} \frac{K_{\mu}^{T}\left(z, z_{0}\right) d m(z)}{\left(\left|z-z_{0}\right| \log \frac{1}{\left|z-z_{0}\right|}\right)^{2}}=o\left(\left[\log \log \frac{1}{\varepsilon}\right]^{2}\right) \quad \forall z_{0} \in \bar{D} .
$$

Here we are able to give a number of other conditions of logarithmic type. In particular, condition (9.3), due to Theorem 9.1, can be replaced by the weaker condition

$$
k_{z_{0}}^{T}(r)=O\left(\log \frac{1}{r} \log \log \frac{1}{r}\right) .
$$

Finally, by Theorem 9.1, applying also Theorem 3.1 in [46], we come to the following result.

Theorem 9.4. Let $D$ be a bounded simply connected domain in $\mathbb{C}$ and let $\mu: D \rightarrow \mathbb{D}$ be a measurable function with $K_{\mu} \in L_{\text {loc }}^{1}$ and

$$
\int_{D \cap B\left(z_{0}, \varepsilon_{0}\right)} \Phi_{z_{0}}\left(K_{\mu}^{T}\left(z, z_{0}\right)\right) d m(z)<\infty \quad \forall z_{0} \in \bar{D}
$$

for $\varepsilon_{0}=\varepsilon\left(z_{0}\right)>0$ and a nondecreasing convex function $\Phi_{z_{0}}:[0, \infty) \rightarrow$ $[0, \infty)$ with

$$
\int_{\delta_{0}}^{\infty} \frac{d \tau}{\tau \Phi_{z_{0}}^{-1}(\tau)}=\infty
$$

for $\delta_{0}=\delta\left(z_{0}\right)>\Phi_{z_{0}}(0)$. Then the Beltrami equation (1.1) has a regular solution $f$ of the Dirichlet problem (1.6) for every continuous function $\varphi: E_{D} \rightarrow \mathbb{R}$.

Remark 9.6. Recall that condition (9.14) is equivalent to each of conditions (7.13)-(7.17). Moreover, condition (9.14) is not only sufficient but also necessary to have a regular solution of the Dirichlet problem (1.6) for every Beltrami equation (1.1) with the integral restriction (9.13) for every continuous function $\varphi: E_{D} \rightarrow \mathbb{R}$. Indeed, by the Stoilow theorem on representation of discrete open mappings, see, e.g., [50], every regular solution $f$ of the Dirichlet problem (1.6) for the Beltrami equation (1.1) with $K_{\mu} \in L_{l o c}^{1}$ can be represented in the form of composition $f=h \circ F$, where $h$ is a holomorphic function and $F$ is a regular homeomorphic solution of (1.1) in the class $W_{\text {loc }}^{1,1}$. Thus, by Theorem 5.1 in [47] on 
the nonexistence of regular homeomorphic solutions of (1.1) in the class $W_{\text {loc }}^{1,1}$, if (9.14) fails, then there is a measurable function $\mu: D \rightarrow \mathbb{D}$ satisfying integral condition (9.13) for which Beltrami equation (1.1) has no regular solution of the Dirichlet problem (1.6) for any nonconstant continuous function $\varphi: E_{D} \rightarrow \mathbb{R}$.

Corollary 9.3. Let $D$ be a bounded simply connected domain in $\mathbb{C}$ and let $\mu: D \rightarrow \mathbb{D}$ be a measurable function with $K_{\mu} \in L_{\text {loc }}^{1}$ and

$$
\int_{D \cap B\left(z_{0}, \varepsilon_{0}\right)} e^{\alpha_{0} K_{\mu}^{T}\left(z, z_{0}\right)} d m(z)<\infty \quad \forall z_{0} \in \bar{D}
$$

for some $\varepsilon_{0}=\varepsilon\left(z_{0}\right)>0$ and $\alpha_{0}=\alpha\left(z_{0}\right)>0$. Then the Beltrami equation (1.1) has a regular solution $f$ of the Dirichlet problem (1.6) for every continuous function $\varphi: E_{D} \rightarrow \mathbb{R}$.

\section{Pseudoregular solutions in multiply connected domains}

As it was probably first noted by B. Bojarski, see, e.g., $\S 6$ of Chapter 4 in [52], the Dirichlet problem for the Beltrami equations, generally speaking, has no regular solution in the class of functions continuous (single-valued) in $\mathbb{C}$ with generalized derivatives in the case of multiply connected domains $D$. Hence, the natural question arises: whether solutions exist in wider classes of functions for this case? It is turned out that solutions for this problem can be found in the class of functions admitting a certain number (related to connectedness of $D$ ) of poles at prescribed points. Later on, this number will take into account the multiplicity of these poles from the Stoilow representation.

A discrete open mapping $f: D \rightarrow \overline{\mathbb{C}}$ of the Sobolev class $W_{\text {loc }}^{1,1}$ (outside of poles) satisfying (1.1) a.e. and the boundary condition (1.6) is called the pseudoregular solution of the Dirichlet problem if the Jacobian $J_{f}(z) \neq 0$ a.e.

Arguing similarly to the case of simply connected domains and applying Theorem V.6.2 in [9] on conformal mappings of finitely connected domains onto circular domains and also Theorems 4.13 and 4.14 in [52], we obtain the following result.

Theorem 10.1. Let $D$ be a bounded $m$-connected domain in $\mathbb{C}$ with nondegenerate boundary components, $k \geqslant m-1$, and let $\mu: D \rightarrow \mathbb{D}$ be a 
measurable function with $K_{\mu} \in L_{\text {loc }}^{1}$ and

$$
\int_{0}^{\delta\left(z_{0}\right)} \frac{d r}{\left\|K_{\mu}^{T}\right\|\left(z_{0}, r\right)}=\infty \quad \forall z_{0} \in \bar{D}
$$

for some $0<\delta\left(z_{0}\right)<d\left(z_{0}\right)=\sup _{z \in D}\left|z-z_{0}\right|$ and

$$
\left\|K_{\mu}^{T}\right\|\left(z_{0}, r\right):=\int_{S\left(z_{0}, r\right)} K_{\mu}^{T}\left(z, z_{0}\right) d s .
$$

Then the Beltrami equation (1.1) has a pseudoregular solution $f$ of the Dirichlet problem (1.6) with $k$ poles at prescribed points in $D$ for every continuous function $\varphi: E_{D} \rightarrow \mathbb{R}$.

Here, as before, we set $K_{\mu}^{T}$ to be extended by zero outside of the domain $D$.

Corollary 10.1. Let $D$ be a bounded $m$-connected domain in $\mathbb{C}$ with nondegenerate boundary components, $k \geqslant m-1$, and let $\mu: D \rightarrow \mathbb{D}$ be a measurable function with $K_{\mu}^{T} \in L_{\mathrm{loc}}^{1}$ and

$$
k_{z_{0}}^{T}(\varepsilon)=O\left(\log \frac{1}{\varepsilon}\right) \quad \text { as } \varepsilon \rightarrow 0 \quad \forall z_{0} \in \bar{D},
$$

where $k_{z_{0}}^{T}(\varepsilon)$ is the average of the function $K_{\mu}^{T}\left(z, z_{0}\right)$ over the circle $S\left(z_{0}, \varepsilon\right)$.

Then the Beltrami equation (1.1) has a pseudoregular solution $f$ of the Dirichlet problem (1.6) with $k$ poles at prescribed points in $D$ for every continuous function $\varphi: E_{D} \rightarrow \mathbb{R}$.

Remark 10.1. In particular, the conclusion of Theorem 10.1 holds if

$$
K_{\mu}^{T}\left(z, z_{0}\right)=O\left(\log \frac{1}{\left|z-z_{0}\right|}\right) \quad \text { as } \quad z \rightarrow z_{0} \quad \forall z_{0} \in \bar{D} .
$$

Proof of Theorem 10.1. Let $F$ be a regular solution of Eq. (1.1) in the class $W_{\text {loc }}^{1,1}$ that exists by condition (10.1), see, e.g., Theorem 5.4 in work [41] or Theorem 11.10 in book [26]. Note that the domain $D^{*}=F(D)$ is $m$-connected in $\overline{\mathbb{C}}$ and there is a natural one-to-one correspondence between components $\gamma_{j}$ of $\gamma=\partial D$ and components $\Gamma_{j}$ of $\Gamma=\partial D^{*}$, $\Gamma_{j}=C\left(\gamma_{j}, F\right)$ and $\gamma_{j}=C\left(\Gamma_{j}, F^{-1}\right), j=1, \ldots, m$, see, e.g., Lemma 5.3 in [14] or Lemma 6.5 in [26]. Moreover, by Remark 1.1, every subspace $E_{j}$ of $E_{D}$ associated with $\gamma_{j}$ consists of more than one prime end, even it is homeomorphic to the unit circle. 
Next, no one of $\Gamma_{j}, j=1, \ldots, m$, is degenerated to a single point. Indeed, let us assume that $\Gamma_{j_{0}}=\left\{\zeta_{0}\right\}$ first for some $\zeta_{0} \in \mathbb{C}$. Let $r_{0} \in$ $\left(0, d_{0}\right)$, where $d_{0}=\inf _{\zeta \in \Gamma \backslash \Gamma_{j_{0}}}\left|\zeta-\zeta_{0}\right|$. Then the punctured disk $D_{0}=$ $\left\{\zeta \in \mathbb{C}: 0<\left|\zeta-\zeta_{0}\right|<r_{0}\right\}$ is in the domain $D^{*}$ and its boundary does not intersect $\Gamma \backslash \Gamma_{j_{0}}$. Set $\widetilde{D}=F^{-1}\left(D_{0}\right)$. Then, by construction, $\widetilde{D} \subset D$ is a 2-connected domain, $\widetilde{D} \cap \gamma \backslash \gamma_{j_{0}}=\varnothing, C\left(\gamma_{j_{0}}, \widetilde{F}\right)=\left\{\zeta_{0}\right\}$ and $C\left(\zeta_{0}, \widetilde{F}^{-1}\right)=\gamma_{j_{0}}$, where $\widetilde{F}$ is a restriction of the mapping $\widetilde{F}$ to $\widetilde{D}$. However, this contradicts Theorem 8.2 because, as was noted above, $E_{j_{0}}$ contains more than one prime end.

Now, let assume that $\Gamma_{j_{0}}=\{\infty\}$. Then the component of $\overline{\mathbb{C}} \backslash D^{*}$ associated with $\Gamma_{j_{0}}$, see Lemma 5.1 in [14] or Lemma 6.3 in [26], is also consists of the single point $\infty$ because if the interior of this component is not empty, then, choosing there an arbitrary point $\zeta_{0}$ and joining it with a point $\zeta_{*} \in D^{*}$ by a segment of a straight line, we would find one more point in $\Gamma_{j_{0}}$, see, e.g., Proposition 2.3 in [36] or Proposition 13.3 in [26].

Thus, applying, if necessary, an additional stretching (conformal mapping), we may assume with no loss of generality that $D^{*}$ contains the exteriority $\mathbb{D}_{*}$ of the unit disk $\mathbb{D}$ in $\mathbb{C}$. Set $\kappa(\zeta)=1 / \zeta, \kappa(0)=\infty, \kappa(\infty)=0$. Consider the mapping $F_{*}=\kappa \circ F: \widetilde{D} \rightarrow \mathbb{D}_{0}$, where $\widetilde{D}=F^{-1}\left(\mathbb{D}_{*}\right)$ and $\mathbb{D}_{0}=\mathbb{D} \backslash\{0\}$ is the punctured unit disk. It is clear that $F_{*}$ is also a homeomorphic solution of the Beltrami equation (1.1) of the class $W_{\text {loc }}^{1,1}$ in a 2-connected domain $\widetilde{D}$ because the mapping $\kappa$ is conformal. Consequently, by Theorem 8.2, elements of $E_{j_{0}}$ should be in a one-to-one correspondence with 0 . However, it was already noted, $E_{j_{0}}$ cannot consists of a single prime end. The obtained contradiction disproves the assumption that $\Gamma_{j_{0}}=\{\infty\}$.

Thus, by Theorem V.6.2 in [9], see also Remark 1.1 in [19], $D^{*}$ can be mapped with a conformal mapping $R_{*}$ onto a bounded circular domain $\mathbb{D}^{*}$ whose boundary consists of mutually disjoint circles. Note that the function $g:=R_{*} \circ F$ is again a regular homeomorphic solution in the Sobolev class $W_{\text {loc }}^{1,1}$ for the Beltrami equation (1.1) that maps $D$ onto $\mathbb{D}^{*}$. By Theorem 8.2, the mapping $g$ admits an extension to a homeomorphism $g_{*}: \bar{D}_{P} \rightarrow \overline{\mathbb{D}^{*}}$.

Let us find a solution of the initial Dirichlet problem (1.6) in the form $f=h \circ g$, where $h$ is a meromorphic function in $\mathbb{D}^{*}$ with the boundary condition

$$
\lim _{z \rightarrow \zeta} \operatorname{Re} h(z)=\varphi\left(g_{*}^{-1}(\zeta)\right) \quad \forall \zeta \in \partial \mathbb{D}^{*}
$$

and $k \geqslant m-1$ poles corresponding under the mapping $g$ to those at prescribed points in $D$. Note that the function from the right-hand side in (10.4) is continuous in the variable $\zeta$. Thus, such a function $h$ 
exists by Theorems 4.13 and 4.14 in [52]. It is clear that the function $f$ associated with $h$ is, by construction, a desired pseudoregular solution of the Dirichlet problem (1.6) for the Beltrami equation (1.1).

Applying Lemma 2.2 in [38], see also Lemma 7.4 in [26], we obtain immediately the next lemma from Theorem 10.1.

Lemma 10.1. Let $D$ be a bounded $m$-connected domain in $\mathbb{C}$ with nondegenerate boundary components, $k \geqslant m-1$, and let $\mu: D \rightarrow \mathbb{D}$ be a measurable function with $K_{\mu} \in L^{1}(D)$. Suppose that, for every $z_{0} \in \bar{D}$ and every small enough $0<\varepsilon_{0}<d\left(z_{0}\right):=\sup _{z \in D}\left|z-z_{0}\right|$, there is a family of measurable functions $\psi_{z_{0}, \varepsilon, \varepsilon_{0}}:(0, \infty) \rightarrow[0, \infty], \varepsilon \in\left(0, \varepsilon_{0}\right)$ such that

$$
0<I_{z_{0}, \varepsilon_{0}}(\varepsilon):=\int_{\varepsilon}^{\varepsilon_{0}} \psi_{z_{0}, \varepsilon, \varepsilon_{0}}(t) d t<\infty \quad \forall \varepsilon \in\left(0, \varepsilon_{0}\right)
$$

and

$\int_{\varepsilon<\left|z-z_{0}\right|<\varepsilon_{0}} K_{\mu}^{T}\left(z, z_{0}\right) \cdot \psi_{z_{0}, \varepsilon, \varepsilon_{0}}^{2}\left(\left|z-z_{0}\right|\right) d m(z)=o\left(I_{z_{0}, \varepsilon_{0}}^{2}(\varepsilon)\right) \quad$ as $\varepsilon \rightarrow 0$.

Then the Beltrami equation (1.1) has a pseudoregular solution $f$ of the Dirichlet problem (1.6) with $k$ poles at prescribed points in $D$ for every continuous function $\varphi: E_{D} \rightarrow \mathbb{R}$.

Remark 10.2. In fact, it is sufficient to assume the local integrability of $K_{\mu}$ in the domain $D$ and the condition $\left\|K_{\mu}\right\|\left(z_{0}, r\right) \neq \infty$ for a.e. $r \in\left(0, \varepsilon_{0}\right)$ and all $z_{0} \in \partial D$ instead of the condition $K_{\mu} \in L^{1}(D)$.

By Lemma 10.1 with the choice $\psi_{z_{0}, \varepsilon}(t) \equiv 1 / t \log \frac{1}{t}$, we obtain the following result, see also Lemma 3.1.

Theorem 10.2. Let $D$ be a bounded $m$-connected domain in $\mathbb{C}$ with nondegenerate boundary components, $k \geqslant m-1$, and let $\mu: D \rightarrow \mathbb{D}$ be a measurable function with $K_{\mu} \in L^{1}(D)$ such that

$$
K_{\mu}^{T}\left(z, z_{0}\right) \leqslant Q_{z_{0}}(z) \in \operatorname{FMO}\left(z_{0}\right) \quad \forall z_{0} \in \bar{D} .
$$

Then the Beltrami equation (1.1) has a pseudoregular solution $f$ of the Dirichlet problem (1.6) with $k$ poles at points in $D$ for every continuous function $\varphi: E_{D} \rightarrow \mathbb{R}$.

Remark 10.3. In particular, the conclusion of Theorem 10.2 holds if either $Q_{z_{0}} \in \mathrm{BMO}_{\text {loc }}$ or $Q_{z_{0}} \in \mathrm{W}_{\text {loc }}^{1,2}$ because $W_{\text {loc }}^{1,2} \subset \mathrm{VMO}_{\text {loc }}$, see, e.g., [3]. 
By Corollary 3.1, we have the next consequence of Theorem 10.2:

Corollary 10.2. Let $D$ be a bounded $m$-connected domain in $\mathbb{C}$ with nondegenerate boundary components, $k \geqslant m-1$, and let $\mu: D \rightarrow \mathbb{D}$ be a measurable function with $K_{\mu} \in L^{1}(D)$ such that

$$
\limsup _{\varepsilon \rightarrow 0} f_{B\left(z_{0}, \varepsilon\right)} K_{\mu}^{T}\left(z, z_{0}\right) d m(z)<\infty \quad \forall z_{0} \in \bar{D} .
$$

Then the Beltrami equation (1.1) has a pseudoregular solution $f$ of the Dirichlet problem (1.6) with $k$ poles at prescribed points in $D$ for every continuous function $\varphi: E_{D} \rightarrow \mathbb{R}$.

Remark 10.4. In particular, by (1.5), the conclusion of Theorem 10.2 holds if

$$
K_{\mu}(z) \leqslant Q(z) \in \operatorname{BMO}(\bar{D}) .
$$

The following statement follows from Lemma 10.1 through the choice $\psi(t)=1 / t$, see also Remark 10.2.

Theorem 10.3. Let $D$ be a bounded m-connected domain in $\mathbb{C}$ with nondegenerate boundary components, $k \geqslant m-1$, and let $\mu: D \rightarrow \mathbb{D}$ be a measurable function such that

$$
\int_{\varepsilon<\left|z-z_{0}\right|<\varepsilon_{0}} K_{\mu}^{T}\left(z, z_{0}\right) \frac{d m(z)}{\left|z-z_{0}\right|^{2}}=o\left(\left[\log \frac{1}{\varepsilon}\right]^{2}\right) \quad \forall z_{0} \in \bar{D} .
$$

Then the Beltrami equation (1.1) has a pseudoregular solution $f$ of the Dirichlet problem (1.6) with $k$ poles at prescribed points in $D$ for every continuous function $\varphi: E_{D} \rightarrow \mathbb{R}$.

Remark 10.5. Similarly, choosing $\psi(t)=1 /(t \log 1 / t)$ instead of $\psi(t)=$ $1 / t$ in Lemma 10.1, we obtain that condition (10.10) can be replaced by the condition

$$
\int_{\varepsilon<\left|z-z_{0}\right|<\varepsilon_{0}} \frac{K_{\mu}^{T}\left(z, z_{0}\right) d m(z)}{\left(\left|z-z_{0}\right| \log \frac{1}{\left|z-z_{0}\right|}\right)^{2}}=o\left(\left[\log \log \frac{1}{\varepsilon}\right]^{2}\right) \quad \forall z_{0} \in \bar{D}
$$

Here we are able to give a number of other conditions of logarithmic type. In particular, condition (10.2), due to Theorem 10.1, can be replaced by the weaker condition

$$
k_{z_{0}}^{T}(r)=O\left(\log \frac{1}{r} \log \log \frac{1}{r}\right) .
$$


Finally, by Theorem 10.1, applying also Theorem 3.1 in work [46], we come to the following result.

Theorem 10.4. Let $D$ be a bounded $m$-connected domain in $\mathbb{C}$ with nondegenerate boundary components, $k \geqslant m-1$, and let $\mu: D \rightarrow \mathbb{D}$ be a measurable function with $K_{\mu} \in L_{l o c}^{1}$ such that

$$
\int_{D \cap B\left(z_{0}, \varepsilon_{0}\right)} \Phi_{z_{0}}\left(K_{\mu}^{T}\left(z, z_{0}\right)\right) d m(z)<\infty
$$

for $\varepsilon_{0}=\varepsilon\left(z_{0}\right)>0$ and a nondecreasing convex function $\Phi_{z_{0}}:[0, \infty) \rightarrow$ $[0, \infty)$ with

$$
\int_{\delta_{0}}^{\infty} \frac{d \tau}{\tau \Phi_{z_{0}}^{-1}(\tau)}=\infty
$$

for $\delta_{0}=\delta\left(z_{0}\right)>\Phi_{z_{0}}(0)$. Then the Beltrami equation (1.1) has a pseudoregular solution $f$ of the Dirichlet problem (1.6) with $k$ poles at prescribed points in $D$ for every continuous function $\varphi: E_{D} \rightarrow \mathbb{R}$.

Recall that condition (10.14) is equivalent to every of conditions (7.13)-(7.17).

Corollary 10.3. Let $D$ be a bounded $m$-connected domain in $\mathbb{C}$ with nondegenerate boundary components, $k \geqslant m-1$, and let $\mu: D \rightarrow \mathbb{D}$ be a measurable function with $K_{\mu} \in L_{\text {loc }}^{1}$ such that

$$
\int_{D \cap B\left(z_{0}, \varepsilon_{0}\right)} e^{\alpha_{0} K_{\mu}^{T}\left(z, z_{0}\right)} d m(z)<\infty \quad \forall z_{0} \in \bar{D}
$$

for some $\varepsilon_{0}=\varepsilon\left(z_{0}\right)>0$ and $\alpha_{0}=\alpha\left(z_{0}\right)>0$.

Then the Beltrami equation (1.1) has a pseudoregular solution $f$ of the Dirichlet problem (1.6) with $k$ poles at prescribed points in $D$ for every continuous function $\varphi: E_{D} \rightarrow \mathbb{R}$.

\section{Multivalent solutions in finitely connected domains}

In finitely connected domains $D$ in $\mathbb{C}$, in addition to pseudoregular solutions, the Dirichlet problem for the Beltrami equation (1.1) admits multivalent solutions in the spirit of the theory of multivalent analytic functions.

We say that a discrete open mapping $f: B\left(z_{0}, \varepsilon_{0}\right) \rightarrow \mathbb{C}$, where $B\left(z_{0}, \varepsilon_{0}\right) \subseteq D$, is a local regular solution of Eq. (1.1) if $f \in W_{\mathrm{loc}}^{1,1}$, 
$J_{f}(z) \neq 0$, and $f$ satisfies $(1.1)$ a.e. in $B\left(z_{0}, \varepsilon_{0}\right)$. The local regular solutions $f: B\left(z_{0}, \varepsilon_{0}\right) \rightarrow \mathbb{C}$ and $f_{*}: B\left(z_{*}, \varepsilon_{*}\right) \rightarrow \mathbb{C}$ of Eq. (1.1) will be called extensions of each to other if there is a finite chain of such solutions $f_{i}$ : $B\left(z_{i}, \varepsilon_{i}\right) \rightarrow \mathbb{C}, i=1, \ldots, m$, that $f_{1}=f_{0}, f_{m}=f_{*}$ and $f_{i}(z) \equiv f_{i+1}(z)$ for $z \in E_{i}:=B\left(z_{i}, \varepsilon_{i}\right) \cap B\left(z_{i+1}, \varepsilon_{i+1}\right) \neq \emptyset, i=1, \ldots, m-1$. A collection of local regular solutions $f_{j}: B\left(z_{j}, \varepsilon_{j}\right) \rightarrow \mathbb{C}, j \in J$, will be called a multivalent solution of Eq. (1.1) in $D$ if the disks $B\left(z_{j}, \varepsilon_{j}\right)$ cover the whole domain $D$ and $f_{j}$ are extensions of each to other through the collection and the collection is maximal by inclusion. A multivalent solution of Eq. (1.1) will be called a multivalent solution of the Dirichlet problem for a prescribed continuous function $\varphi: E_{D} \rightarrow \mathbb{R}$ if $u(z)=\operatorname{Re} f(z)=\operatorname{Re} f_{j}(z)$, $z \in B\left(z_{j}, \varepsilon_{j}\right), j \in J$, is a single-valued function in $D$ satisfying the condition $\lim _{z \rightarrow P} u(z)=\varphi(P)$ along any ways in $D$ going to $P \in E_{D}$.

As above, we assume later on that $K_{\mu}^{T}\left(\cdot, z_{0}\right)$ is extended by zero outside of the domain $D$.

The proof of the existence of multivalent solutions of the Dirichlet problem (1.6) for the Beltrami equation (1.1) in finitely connected domains is reduced on the basis of Section 8 to the Dirichlet problem for harmonic functions in circular domains, see, e.g., §3 of Chapter VI in [9].

Theorem 11.1. Let $D$ be a bounded finitely connected domain in $\mathbb{C}$ with nondegenerate boundary components and let $\mu: D \rightarrow \mathbb{D}$ be a measurable function with $K_{\mu} \in L_{\mathrm{loc}}^{1}$ and

$$
\int_{0}^{\delta\left(z_{0}\right)} \frac{d r}{\left\|K_{\mu}^{T}\right\|\left(z_{0}, r\right)}=\infty \quad \forall z_{0} \in \bar{D}
$$

for some $0<\delta\left(z_{0}\right)<d\left(z_{0}\right)=\sup _{z \in D}\left|z-z_{0}\right|$ and

$$
\left\|K_{\mu}^{T}\right\|\left(z_{0}, r\right):=\int_{S\left(z_{0}, r\right)} K_{\mu}^{T}\left(z, z_{0}\right) d s
$$

Then the Beltrami equation (1.1) has a multivalent solution of the Dirichlet problem (1.6) for every continuous function $\varphi: E_{D} \rightarrow \mathbb{R}$.

Proof of Theorem 11.1. Similarly to the first part of Theorem 10.1, it is proved that there is a regular homeomorphic solution $g$ of the Beltrami equation (1.1) mapping the domain $D$ onto a circular domain $\mathbb{D}^{*}$ whose boundary consists of mutually disjoint circles. By Theorem 8.2, the mapping $g$ admits an extension to a homeomorphism $g_{*}: \bar{D}_{P} \rightarrow \overline{\mathbb{D}}^{*}$. 
As known, in the circular domain $\mathbb{D}^{*}$, there is a solution of the Dirichlet problem

$$
\lim _{z \rightarrow \zeta} u(z)=\varphi\left(g_{*}^{-1}(\zeta)\right) \quad \forall \zeta \in \partial \mathbb{D}^{*}
$$

for harmonic functions $u$, see, e.g., $\S 3$ of Chapter VI in [9]. Let $B_{0}=$ $B\left(z_{0}, r_{0}\right)$ be a disk in the domain $D$. Then $\mathfrak{B}_{0}=g\left(B_{0}\right)$ is a simply connected subdomain of the circular domain $\mathbb{D}^{*}$, where there is a conjugate function $v$ determined up to an additive constant such that $h=u+i v$ is a single-valued analytic function. The function $h$ can be extended to, generally speaking, a multivalent analytic function $H$ along any path in $\mathbb{D}^{*}$ because $u$ is given in the whole domain $\mathbb{D}^{*}$.

Thus, $f=H \circ g$ is a desired multivalent solution of the Dirichlet problem (1.6) for the Beltrami equation (1.1).

The hypotheses of the rest theorems and corollaries below yield the hypotheses of Theorem 11.1, as was shown in the previous section.

Corollary 11.1. Let $D$ be a bounded finitely connected domain in $\mathbb{C}$ with nondegenerate boundary components and let $\mu: D \rightarrow \mathbb{D}$ be a measurable function with $K_{\mu} \in L_{\text {loc }}^{1}$ and

$$
k_{z_{0}}^{T}(\varepsilon)=O\left(\log \frac{1}{\varepsilon}\right) \quad \text { as } \quad \varepsilon \rightarrow 0 \quad \forall z_{0} \in \bar{D},
$$

where $k_{z_{0}}^{T}(\varepsilon)$ is the average of the function $K_{\mu}^{T}\left(z, z_{0}\right)$ over the circle $S\left(z_{0}, \varepsilon\right)$.

Then the Beltrami equation (1.1) has a multivalent solution of the Dirichlet problem (1.6) for every continuous function $\varphi: E_{D} \rightarrow \mathbb{R}$.

Remark 11.1. In particular, the conclusion of Theorem 11.1 holds if

$$
K_{\mu}^{T}\left(z, z_{0}\right)=O\left(\log \frac{1}{\left|z-z_{0}\right|}\right) \quad \text { as } \quad z \rightarrow z_{0} \quad \forall z_{0} \in \bar{D} .
$$

Applying Lemma 2.2 in [38], see also Lemma 7.4 in [26], we obtain the following result immediately from Theorem 11.1.

Lemma 11.1. Let $D$ be a bounded finitely connected domain in $\mathbb{C}$ with nondegenerate boundary components and let $\mu: D \rightarrow \mathbb{D}$ be a measurable function with $K_{\mu} \in L^{1}(D)$. Suppose that, for every $z_{0} \in \bar{D}$ and every small enough $0<\varepsilon_{0}<d\left(z_{0}\right):=\sup _{z \in D}\left|z-z_{0}\right|$, there is a family of measurable functions $\psi_{z_{0}, \varepsilon, \varepsilon_{0}}:(0, \infty) \rightarrow[0, \infty], \varepsilon \in\left(0, \varepsilon_{0}\right)$ such that

$$
0<I_{z_{0}, \varepsilon_{0}}(\varepsilon):=\int_{\varepsilon}^{\varepsilon_{0}} \psi_{z_{0}, \varepsilon, \varepsilon_{0}}(t) d t<\infty \quad \forall \varepsilon \in\left(0, \varepsilon_{0}\right)
$$


and

$\int_{\varepsilon<\left|z-z_{0}\right|<\varepsilon_{0}} K_{\mu}^{T}\left(z, z_{0}\right) \cdot \psi_{z_{0}, \varepsilon, \varepsilon_{0}}^{2}\left(\left|z-z_{0}\right|\right) d m(z)=o\left(I_{z_{0}, \varepsilon_{0}}^{2}(\varepsilon)\right) \quad$ as $\varepsilon \rightarrow 0$.

Then the Beltrami equation (1.1) has a multivalent solution of the Dirichlet problem (1.6) for every continuous function $\varphi: E_{D} \rightarrow \mathbb{R}$.

Remark 11.2. In fact, it is sufficient to assume the local integrability of $K_{\mu}^{T}$ in the domain $D$ and the condition $\left\|K_{\mu}^{T}\right\|\left(z_{0}, r\right) \neq \infty$ for a.e. $r \in\left(0, \varepsilon_{0}\right)$ and all $z_{0} \in \partial D$ instead of the condition $K_{\mu}^{T} \in L^{1}(D)$.

By Lemma 11.1 with the choice $\psi_{z_{0}, \varepsilon}(t) \equiv 1 / t \log \frac{1}{t}$, we obtain the following result, see also Lemma 3.1.

Theorem 11.2. Let $D$ be a bounded finitely connected domain in $\mathbb{C}$ with nondegenerate boundary components and let $\mu: D \rightarrow \mathbb{D}$ be a measurable function with $K_{\mu} \in L^{1}(D)$ such that

$$
K_{\mu}^{T}\left(z, z_{0}\right) \leqslant Q_{z_{0}}(z) \in \operatorname{FMO}\left(z_{0}\right) \quad \forall z_{0} \in \bar{D}
$$

Then the Beltrami equation (1.1) has a multivalent solution of the Dirichlet problem (1.6) for every continuous function $\varphi: E_{D} \rightarrow \mathbb{R}$.

Remark 11.3. In particular, the conclusion of Theorem 11.2 holds if either $Q_{z_{0}} \in \mathrm{BMO}_{\text {loc }}$ or $Q_{z_{0}} \in \mathrm{W}_{\text {loc }}^{1,2}$ because $W_{\text {loc }}^{1,2} \subset \mathrm{VMO}_{\text {loc }}$, see, e.g., $[3]$.

By Corollary 3.1, we have the next consequence of Theorem 11.2:

Corollary 11.2. Let $D$ be a bounded finitely connected domain in $\mathbb{C}$ with nondegenerate boundary components and let $\mu: D \rightarrow \mathbb{D}$ be a measurable function with $K_{\mu} \in L^{1}(D)$ such that

$$
\limsup _{\varepsilon \rightarrow 0} f_{B\left(z_{0}, \varepsilon\right)} K_{\mu}^{T}\left(z, z_{0}\right) d m(z)<\infty \quad \forall z_{0} \in \bar{D}
$$

Then the Beltrami equation (1.1) has a multivalent solution of the Dirichlet problem (1.6) for every continuous function $\varphi: E_{D} \rightarrow \mathbb{R}$.

Remark 11.4. In particular, by (1.5), the conclusion of Theorem 11.2 holds if

$$
K_{\mu}(z) \leqslant Q(z) \in \operatorname{BMO}(\bar{D})
$$


The following statement follows from Lemma 11.1 through the choice $\psi(t)=1 / t$, see also Remark 11.2.

Theorem 11.3. Let $D$ be a bounded finitely connected domain in $\mathbb{C}$ with nondegenerate boundary components and let $\mu: D \rightarrow \mathbb{D}$ be a measurable function such that

$$
\int_{\varepsilon<\left|z-z_{0}\right|<\varepsilon_{0}} K_{\mu}^{T}\left(z, z_{0}\right) \frac{d m(z)}{\left|z-z_{0}\right|^{2}}=o\left(\left[\log \frac{1}{\varepsilon}\right]^{2}\right) \quad \forall z_{0} \in \bar{D} .
$$

Then the Beltrami equation (1.1) has a multivalent solution of the Dirichlet problem (1.6) for every continuous function $\varphi: E_{D} \rightarrow \mathbb{R}$.

Remark 11.5. Similarly, $\psi(t)=1 /(t \log 1 / t)$ instead of $\psi(t)=1 / t$ choosing in Lemma 11.1, we obtain that condition (11.10) can be replaced by the condition

$$
\int_{\varepsilon<\left|z-z_{0}\right|<\varepsilon_{0}} \frac{K_{\mu}^{T}\left(z, z_{0}\right) d m(z)}{\left(\left|z-z_{0}\right| \log \frac{1}{\left|z-z_{0}\right|}\right)^{2}}=o\left(\left[\log \log \frac{1}{\varepsilon}\right]^{2}\right) \quad \forall z_{0} \in \bar{D} .
$$

Here we are able to give a number of other conditions of logarithmic type. In particular, condition (11.3), due to Theorem 11.1, can be replaced by the weaker condition

$$
k_{z_{0}}^{T}(r)=O\left(\log \frac{1}{r} \log \log \frac{1}{r}\right) .
$$

Finally, by Theorem 11.1, applying also Theorem 3.1 in work [46], we come to the following result.

Theorem 11.4. Let $D$ be a bounded finitely connected domain in $\mathbb{C}$ with nondegenerate boundary components and let $\mu: D \rightarrow \mathbb{D}$ be a measurable function with $K_{\mu} \in L_{\text {loc }}^{1}$ such that

$$
\int_{D \cap B\left(z_{0}, \varepsilon_{0}\right)} \Phi_{z_{0}}\left(K_{\mu}^{T}\left(z, z_{0}\right)\right) d m(z)<\infty
$$

for $\varepsilon_{0}=\varepsilon\left(z_{0}\right)>0$ and a nondecreasing convex function $\Phi_{z_{0}}:[0, \infty) \rightarrow$ $[0, \infty)$ with

$$
\int_{\delta_{0}}^{\infty} \frac{d \tau}{\tau \Phi_{z_{0}}^{-1}(\tau)}=\infty
$$

for $\delta_{0}=\delta\left(z_{0}\right)>\Phi_{z_{0}}(0)$. Then the Beltrami equation (1.1) has a multivalent solution of the Dirichlet problem (1.6) for every continuous function $\varphi: E_{D} \rightarrow \mathbb{R}$. 
Recall that condition (11.14) is equivalent to every of conditions $(7.13)-(7.17)$.

Corollary 11.3. Let $D$ be a bounded finitely connected domain in $\mathbb{C}$ with nondegenerate boundary components and let $\mu: D \rightarrow \mathbb{D}$ be a measurable function with $K_{\mu} \in L_{\text {loc }}^{1}$ such that

$$
\int_{D \cap B\left(z_{0}, \varepsilon_{0}\right)} e^{\alpha_{0} K_{\mu}^{T}\left(z, z_{0}\right)} d m(z)<\infty \quad \forall z_{0} \in \bar{D}
$$

for some $\varepsilon_{0}=\varepsilon\left(z_{0}\right)>0$ and $\alpha_{0}=\alpha\left(z_{0}\right)>0$.

Then the Beltrami equation (1.1) has a multivalent solution of the Dirichlet problem (1.6) for every continuous function $\varphi: E_{D} \rightarrow \mathbb{R}$.

\section{References}

[1] C. Andreian Cazacu, On the length-area dilatation // Complex Var. Theory Appl., 50 (2005), No. 7-11, 765-776.

[2] B. Bojarski, Generalized solutions of a system of differential equations of the first order of the elliptic type with discontinuous coefficients // Mat. Sb., 43(85) (1957), No. 4, 451-503.

[3] H. Brezis, L. Nirenberg, Degree theory and BMO. I. Compact manifolds without boundaries // Selecta Math. (N.S.), 1 (1995), No. 2, 197-263.

[4] C. Carathéodory, Über die Begrenzung der einfachzusammenhängender Gebiete // Math. Ann., 73 (1913), 323-370.

[5] E. F. Collingwood, A. J. Lohwater, The Theory of Cluster Sets, Cambridge Tracts in Math. and Math. Physics, 56, Cambridge: Cambridge Univ. Press, 1966.

[6] F. Chiarenza, M. Frasca, P. Longo, $W^{2, p}$-solvability of the Dirichlet problem for nondivergence elliptic equations with VMO coefficients // Trans. Amer. Math. Soc., 336 (1993), No. 2, 841-853.

[7] F. W. Gehring, Rings and quasiconformal mappings in space // Trans. Amer. Math. Soc., 103 (1962), 353-393.

[8] F. W. Gehring, O. Martio, Quasiextremal distance domains and extension of quasiconformal mappings // J. Anal. Math. 45 (1985), 181-206.

[9] G. M. Goluzin, Geometric Theory of Functions of a Complex Variable, Transl. of Math. Monographs 26, Providence, RI: AMS, 1969.

[10] V. Gutlyanskii, O. Martio, T. Sugawa, M. Vuorinen, On the degenerate Beltrami equation // Trans. Amer. Math. Soc., 357 (2005), 875-900.

[11] V. Gutlyanskii, V. Ryazanov, U. Srebro, and E. Yakubov, The Beltrami Equations: A Geometric Approach, Developments in Math. 26, New York: Springer, 2012.

[12] J. Heinonen, T. Kilpelainen, O. Martio, Nonlinear Potential Theory of Degenerate Elliptic Equations, Oxford: Clarendon Press, 1993.

[13] A. Hurwitz, R. Courant, The Function theory, Nauka, Moscow, 1968 [in Russian]. 
[14] A. A. Ignat'ev, V. I. Ryazanov, Finite mean oscillation in the mapping theory // Ukrainian Math. Bull., 2 (2005), No. 3, 403-424.

[15] T. Iwaniec, C. Sbordone, Riesz transforms and elliptic PDEs with VMO coefficients // J. Anal. Math., 74 (1998), 183-212.

[16] F. John, L. Nirenberg, On functions of bounded mean oscillation // Comm. Pure Appl. Math., 14 (1961), 415-426.

[17] D. Kovtonyuk, I. Petkov, V. Ryazanov, On the boundary behaviour of solutions to the Beltrami equations // Complex Variables and Elliptic Equations, 58 (2013), No. 5, 647-663.

[18] D. A. Kovtonyuk, I. V. Petkov, V. I. Ryazanov, R. R. Salimov, Boundary behavior and Dirichlet problem for Beltrami equations // Algebra and Analysis, 25 (2013), No. 4, 101-124 [in Russian]; transl in St. Petersburg Math. J., 25 (2014), 587-603.

[19] D. Kovtonyuk, I. Petkov, V. Ryazanov, R. Salimov, On the Dirichlet problem for the Beltrami equation // J. Anal. Math., 122 (2014), No. 4, 113-141.

[20] D. Kovtonyuk, V. Ryazanov, To the theory of boundaries of space domains // Proc. Inst. Appl. Math. \& Mech. NAS of Ukraine, 13 (2006), 110-120 [in Russian].

[21] D. Kovtonyuk, V. Ryazanov, On the theory of lower Q-homeomorphisms // Ukr. Mat. Visn., 5 (2008), No. 2, 159-184; transl. in Ukrain. Math. Bull., 5 (2008), No. 2, 157-181.

[22] D. Kovtonyuk, V. Ryazanov, On the boundary behavior of generalized quasiisometries // J. Anal. Math., 115 (2011), 103-120.

[23] O. Lehto, Homeomorphisms with a prescribed dilatation // Lecture Notes in Math., 118 (1968), 58-73.

[24] O. Martio, V. Ryazanov, U. Srebro, E. Yakubov, Q-homeomorphisms // Contemporary Math., 364 (2004), 193-203.

[25] O. Martio, V. Ryazanov, U. Srebro, E. Yakubov, On Q-homeomorphisms // Ann. Acad. Sci. Fenn. Ser. A1 Math., 30 (2005), 49-69.

[26] O. Martio, V. Ryazanov, U. Srebro, E. Yakubov, Moduli in Modern Mapping Theory, New York: Springer, 2009.

[27] O. Martio, V. Ryazanov, M. Vuorinen, BMO and Injectivity of Space Quasiregular Mappings // Math. Nachr., 205 (1999), 149-161.

[28] O. Martio, J. Sarvas, Injectivity theorems in plane and space // Ann. Acad. Sci. Fenn. Ser. A1 Math., 4 (1978/1979), 384-401.

[29] R. Näkki, Boundary behavior of quasiconformal mappings in n-space // Ann. Acad. Sci. Fenn. Ser. A1 Math., 484 (1970), 1-50.

[30] R. Näkki, Extension of Loewner's capacity theorem // Trans. Amer. Math. Soc., 180, 229-236 (1973).

[31] R. Näkki, Prime ends and quasiconformal mappings // J. Anal. Math., 35 (1979), $13-40$.

[32] D. K. Palagachev, Quasilinear elliptic equations with VMO coefficients // Trans. Amer. Math. Soc., 347 (1995), No. 7, 2481-2493.

[33] M. A. Ragusa, Elliptic boundary value problem in vanishing mean oscillation hypothesis // Comment. Math. Univ. Carolin., 40 (1999), No. 4, 651-663.

[34] E. Reich, H. Walczak, On the behavior of quasiconformal mappings at a point // Trans. Amer. Math. Soc., 117 (1965), 338-351. 
[35] H. M. Reimann, T. Rychener, Funktionen Beschränkter Mittlerer Oscillation // Lecture Notes in Math., 487 (1975).

[36] V. Ryazanov, R. Salimov, Weakly flat spaces and boundaries in the mapping theory // Ukr. Mat. Visn., 4 (2007), No. 2, 199-234; transl. in Ukrain. Math. Bull. 4 (2007), No. 2, 199-233.

[37] V. Ryazanov, R. Salimov, U. Srebro, E. Yakubov, On Boundary Value Problems for the Beltrami Equations // Contemporary Math., 591 (2013), 211-242.

[38] V. Ryazanov, E. Sevost'yanov, Equicontinuons classes of ring Qhomeomorphisms // Sibirsk. Mat. Zh., 48 (2007), No. 6, 1361-1376 [in Russian]; transl. in Siberian Math. J., 48 (2007), No. 6, 1093-1105.

[39] V. Ryazanov, E. Sevost'yanov, Equicontinuity of mappings quasiconformal in the mean // Ann. Acad. Sci. Fenn., 36 (2011), 231-244.

[40] V. Ryazanov, U. Srebro, E. Yakubov, BMO-quasiconformal mappings // J. Anal. Math., 83 (2001), 1-20.

[41] V. Ryazanov, U. Srebro, E. Yakubov, On ring solutions of Beltrami equation // J. Anal. Math., 96 (2005), 117-150.

[42] V. Ryazanov, U. Srebro, E. Yakubov, Beltrami equation and FMO functions // Contemp. Math., 382 (2005), 357-364.

[43] V. Ryazanov, U. Srebro, E. Yakubov, Finite mean oscillation and the Beltrami equation // Israel J. Math., 153 (2006), 247-266.

[44] V. Ryazanov, U. Srebro, E. Yakubov, To strong ring solutions of the Beltrami equations // Uzbek. Math. J., (2009), No. 1, 127-137.

[45] V. Ryazanov, U. Srebro, E. Yakubov, On strong solutions of the Beltrami equations // Complex Var. Elliptic Equ., 55 (2010), No. 1-3, 219-236.

[46] V. Ryazanov, U. Srebro, E. Yakubov, Integral conditions in the mapping theory // Ukr. Mat. Visn., 7 (2010), No. 1, 73-87; transl. in Math. Sci. J., 173 (2011), No. 4, 397-407.

[47] V. Ryazanov, U. Srebro, E. Yakubov, Integral conditions in the theory of the Beltrami equations // Complex Var. Elliptic Equ., 57 (2012), No. 12, 1247-1270.

[48] S. Saks, Theory of the Integral, New York: Dover, 1964.

[49] D. Sarason, Functions of vanishing mean oscillation // Trans. Amer. Math. Soc., 207 (1975), 391-405.

[50] S. Stoilow, Lecons sur les Principes Topologue de le Theorie des Fonctions Analytique, Paris: Gauthier-Villars, 1938.

[51] J. Väisälä, Lectures on n-Dimensional Quasiconformal Mappings, Lecture Notes in Math., 229. Berlin: Springer, 1971.

[52] I. N. Vekua, Generalized analytic functions, London: Pergamon Press, 1962.

[53] R. L. Wilder, Topology of Manifolds, New York: AMS, 1949. 
Contact information

Vladimir

Gutlyanskii, Institute of Applied Mathematics Vladimir Ryazanov and Mechanics, NAS of Ukraine, 84100 Slavyansk, Dondass region, Ukraine

E-Mail: vladimirgut@mail.ru, vlryazanov1@rambler.ru, vl_ryazanov1@mail.ru

Eduard Yakubov

Holon Institute of Technology, Holon, Israel E-Mail: yakubov@hit.ac.il, eduardyakubov@gmail.com 